Division of Geological \& Geophysical Surveys

RAW-DATA FILE 2000-2

\title{
MAJOR OXIDE, MINOR OXIDE, TRACE ELEMENT, AND GEOCHEMICAL DATA FROM ROCKS COLLECTED IN THE IRON CREEK AREA, TALKEETNA MOUNTAINS B-5 QUADRANGLE, ALASKA IN 1999
}

by

M.B. Werdon. J.R. Riehle, J.M. Schmidr. R.J. Newberry, and G.H. Pessel

$\$ 13.00$

May 2000

THIS REPORT HAS NOT BEEN REVIEWED GOR TECHNICAL CONTENT OR FOR CONFORMITY TO THE EDITORIAL STANDARDS OF DGGS

Released by

STATE OF ALASKA

DEPARTMENT OF NATURAL RESOURCES

Division of Geological \& Geophysical Surveys

794 University Avenue, Suite 200

Fairbanks, Alaska 99709-3645 
INTRODUCTION

ANALY'TICAL METHODS

\section{TABLES}

TABLE I. LOCATION AND DESCRIPTION OF ROCK SAMPEES COLLECTED IN THE IRON CREEK AREA FOR TRACE ELEMENT GEOCHEMICAL ANALYSES.

TABLE 2. CONCENTRATION OF TRACE ELEMENTS IN ROCK SAMPLES COLLECTED IN THE IRON CREEK AREA.

TABLE 3. LOCATION AND DESCRIPTION OF ROCK SAMPLES COLLECTED IN THE IRON CREEK AREA FOR MAJOR OXIDE. MINOR OXIDE, AND TRACE ELEMENT ANALYSES.

TABLE + . CONCENTRATION OF MAJOR OXIDES, MINOR OXIDES, AND TRACE ELEMENTS IN ROCK SAMPLES COLLECTED THE IRON CREEX AREA.

TABLE 5. DETECTION LIMITS FOR TRACE-ELEMENT GEOCHEMICAL ANALYSES

TABLE 6. DETECTION LIMITS FOR " $83 \mathrm{Hn}$ " GEOCHEMICAL ANALYSES .

TABLE 7. DETECTION LIMITS FOR MAJOR-OXIDE, MINOR-OXIDE, AND TRACE ELEMENT ANALYSES

\section{SHEETS}

SHEET 1. LOCATION MAP OF ROCK SAMPLES ANALYZED FOR MAJOR-OXIDES, IRON CREEK AREA. TALKEETNA MOUNTAINS B-5 QUADRANGLE, ALASKA IN 1999, 1:63.360 SCAlE, I SHEET (IN POCKET)

SHEET 2, LOCATION MAP OF ROCK SAMPLES ANALYZED FOR TRACE ELEMENT GEOCHEMISTRY, IRON CREEK AREA. TALKEETNA MOUNTAINS B-S QUADRANGLE, ALASKA IN 1999. 1:63,360 SCALE, I SHEET (IN POCKET)

Note: This report (including all analytical data, tables, and map sheets) is available in digital format from the DGGS web site (http://wwwdggs.dnr.state.ak.us) at no charge. The digital data are available as PDF files and Excel spreadsheets. 


\title{
MAJOR OXIDE, MINOR OXIDE. TRACE ELEMENT, AND GEOCHEMICAL DATA FROM ROCKS COLLECTED IN THE IRON CREEK AREA, TALKEETNA MOUNTAINS B-5 QUADRANGLE, ALASKA IN 1999
}

\author{
by
}

\author{
M.B. WERDON, J.R. RIEHLE. J.M. SCHMIDT, R.J. NEWBERRY, and G.H. PESSEL \\ INTRODUCTION
}

Mineral resource personnel from the Alaska Division of Geological \& Geophysical Surveys and the U.S. Geological Survey carried out a joint geological field survey, including mapping and sampling of the Iron Creek area in the southeast Talkeetna Mountains B.5 quadrangle, Alaska from July 11-25, 1999. The fieldwork provides basic information critical to building an understanding of Alaska's geology and is pan of an integrated program of airborne geophysical surveys followed by geological mapping programs. During 1999, 87 rock samples were collected for geochemical rrace-element analysis, and 88 samples were collected for whole rock (major and minor oxides, and petrogenetically important trace element data) analysis. 26 geochemical trace-element samples collected in 1983 are also repored in this study. The locations of these samples are shown on Sheets 1 (whole rock) and 2 (trace-element geochemistry). Location data (in UTM coordinates with a Clark 1866. NAD27, UTM zone 6 projection), descriptions, and analytical results for each sample are cabulated in Tables 1, 2, 3, and 4.

\section{ANALYTICAL METHODS}

All 1999 trace-element geochemical analyses were performed by Chemex Labs, Inc. Rock samples were crushed so that at least 70 percent of the material passed through a -10 (2 mm) mesh screen. A 200 -gram, representative splis of the sample was then taken using a riffle splitter. The 200-gram sample was then pulverized in a chrome steel ring mill so that 95 percent of the sample passed through a -150 (106 micron) mesh screen.

For the samples collected in 1999, gold was analyzed on a 30 gram representative sample split using Fire Assay (FA) and Acomic Absorprion Spectroscopy (AAS) methods. Most of the trace elements were analyzed by Inductively Coupled Plasma - Atomic Emission Spectroscopy (ICP-AES) methods after nitric aqua regia digestion. All $S_{n}$ analyses were determined by the AAS method. Sampies containing greater than l percent Cu were furher

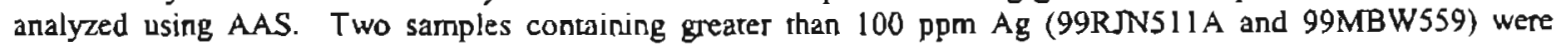
further analyzed using FA-AAS (Fire Assay - Atomic Absorption Spectroscopy). Samples containing greater than or equal to $1 \mathrm{ppm} \mathrm{Hg}$ (by the ICP-AES method) were further analyzed using AAS. Since samples containing greater than 10,000 ppm copper have interference between the specrral lines of copper and bismuth in ICP-AES analyses. where possible, the pulps for these samples were re-run for bismuth using AAS. Analytical detection limits are tabulated in Table S. One sample (99Arj044B) was analyzed by Inductively Coupled Plasma - Mass Spectrometry (ICP-MS) and in Table 5 the elements analyzed by this method are noted by a single asterisk (*).

For the 1983 samples (prefixed by $83 \mathrm{Hn}$ ), $\mathrm{Au}, \mathrm{Ba}, \mathrm{W}$, and $\mathrm{Hg}$ were analyzed by Chemex Labs, Inc. Au and $\mathrm{Hg}$ were analyzed by the AAS method, $W$ was anaiyzed by a colormetric technique, and $B$ a was analyzed by $X$-Ray Fluorescence (XRF). Ag, As, $\mathrm{Co}, \mathrm{Cu}, \mathrm{Mn}, \mathrm{Mo}, \mathrm{Ni}, \mathrm{Pb}, \mathrm{Sb}$, and $\mathrm{Zn}$ were analyzed by the Alaska Division of Geological \& Geophysical Surveys analytical lab by the AAS method after an aqua-regia leach. Analytical detection limits are tabulated in Table 6. The pulps from the 1983 samples were also analyzed for Sn and Bi in 1999 by Chemex Labs, Inc. by the methods described above.

All whole rock analyses were performed by Chemex Labs, Inc. Major and minor element oxides were determined by XRF methods following a metaborace fusion. Trace elements ( $\mathrm{Ba}, \mathrm{Nb}, \mathrm{Rb}, \mathrm{Sr}, \mathrm{Y}$, and $\mathrm{Zr}$ ) were also analyzed using XRF methods on a pressed pellet. A subset of the samples was also analyzed for $\mathrm{Cu}$ and Ni using AAS following an aqua-regia leach, and $\mathrm{Cr}$ using AAS following $\mathrm{HClO}_{4} / \mathrm{HNO}_{3} / \mathrm{HF}$ total digestion. Analyrical detection limits are tabulated in Table 7. 
Table I. Location and description of rock samples collected in the Iron Creek area for trace element geochemical analyses.

\begin{tabular}{|c|c|c|c|}
\hline Sample & UTME & UTMN & Description \\
\hline 99ARJ029 & 387241 & 6911484 & $\begin{array}{l}\text { Weak gossan on hilside with abundant disseminated pyrhotile in a hornfelsed and fractured, medium gray, } \\
\text { very fine grained metavolcanic(?) rock. }\end{array}$ \\
\hline 99AR.J038A & 393843 & 6920011 & $\begin{array}{l}\text { Medium gray, finely porphyritic (feidspar phenocrysts), snallow intrusive intermediate(?) rock cut by } 2-10 \text { 3-mm- } \\
\text { wide vein of quartz and pyrite (partially altered to limonite). }\end{array}$ \\
\hline 99ARJ069 & 391822 & 6913512 & $\begin{array}{l}\text { Medium greenish gray, aphanitic to faintly crystalline(?). amygdaloidal, intermediate to mafic rock with trace } \\
\text { disseminated chalcopyrite and one 1-mm-wide vein of quartz + chalcopyrite + malachite + epidote + maroon } \\
\text { hematite. }\end{array}$ \\
\hline 99PE010A & 392736 & 6913564 & $\begin{array}{l}\text { Small shear zone ( }-2 \text { feel wide) with quartz + magnetite veins in copper-stained greenstone with disseminated } \\
\text { suffides. }\end{array}$ \\
\hline$\overline{9} \overline{9}$ PE63 & 393161 & 6914064 & 1 foot wide, rusty weathering quartz vein with pyrite in epidote altered and veined greenstone. \\
\hline 99RN525 & 394590 & 6916163 & $\begin{array}{l}\text { Float of nearly massive hematite + quartz material (up to } 25 \mathrm{~cm} \text { in diameler) mixed with amygdaloidal } \\
\text { greenstone. }\end{array}$ \\
\hline 99JS29C & 395648 & 6914721 & Brick-red weathering, very dark gray siliceous rock with very fine grained disseminated pyrite. \\
\hline 99JS33C & 393603 & 6922036 & Very dark red-brown-orange stained area in metabasatt. \\
\hline 99JS40B & 395639 & 6920792 & Red-brown weathering material on fracture surfaces in fine grained diorite or microgabbro. \\
\hline 99JS46A & 393641 & 6913728 & $\begin{array}{l}\text { Dark brown to black-red weathering, epidole altered, amygdaloidal, hematite(?)-bearing lava with abundant } \\
\text { malachite staining. }\end{array}$ \\
\hline 99JS58A & 384522 & 6910814 & $\begin{array}{l}\text { Very bright red-orange stained zone ( } 100 \text { feet by } 100 \text { feet), with red stained rocks contairing epidote, quartz, } \\
\text { specular hematite, and trace copper carbonate. }\end{array}$ \\
\hline 99MBW537 & 395694 & 6915760 & $\begin{array}{l}25 \text { foot by } 10 \text { fool trench in intensely iron stained, quartz veined, greenstone with abundant disseminated pyrite } \\
\text { and possibly chalcopyrite. Veins contain chlorite, earthy and specular hematte, quartz, and chalcopyrite. } \\
\text { Chalcopyrite is spatially assoclated with quartz-rich areas in the veins. }\end{array}$ \\
\hline $99 M B W 515 B$ & $395 \uparrow 15$ & 6912440 & $\begin{array}{l}\text { Very iron-stained, orange weathering, gray siliceous rock with up to } 40 \% \text { disseminated pyrite, }+/ \text { - cut by quartz } \\
\text { velns. Possible mafic host rock in a hornfelsed zone above a quartz diorite pluton. }\end{array}$ \\
\hline $99 \mathrm{MBW} 430 \mathrm{~B}$ & 395194 & 6914763 & $\begin{array}{l}\text { Small iron stained gossan zone } \sim 3 \text { feet wide with quartz, calcite, pyrite, chalcopyrite, malachite, and limonite. } \\
\text { The zone trends at an azimuth of }-45+1-10 \text { degrees across the ridge. }\end{array}$ \\
\hline $99 \mathrm{MBW} 515$ & 395115 & 6912440 & $\begin{array}{l}\text { Very iron-stained, orange weathering, gray siliceous rock with up to } 40 \% \text { disseminated pyrite, }+/ \text { - cut by quartz } \\
\text { veins. Possible mafic host rock in a hornfelsed zone above the quartz diorite pluton. }\end{array}$ \\
\hline 99ARJJ030B & 393189 & 6913124 & Massive milky quariz vein intergrown with white calcite. \\
\hline & 393843 & 6920011 & $\begin{array}{l}\text { Large iron-stained quartz vein. The quartz is irregularly intergrown with dark greenist-black, very fine grained } \\
\text { chlorite(?) and (or) hematite(?), and also contains trace disseminated pyrite. }\end{array}$ \\
\hline 99ARJ068 & 394804 & $69177+9$ & Grayish green, amygdaloidal, epidote allered microgabbro cut by quartz veins. \\
\hline
\end{tabular}


Table 1. Location and description of rock samples collected in the Iron Creek area for trace element geochemical analyses.

\begin{tabular}{|c|c|c|c|}
\hline Sample & UTME & UTM N & Description \\
\hline 99PE010B & 392736 & 6913564 & $\begin{array}{l}\text { Recessive zone of greenstone with common linear veins that contain quartz and sulfides; non-magnetic. } \\
\text { Fractures and veins are about } 0.1-2 \mathrm{~cm} \text { wide with abundant copper staining. }\end{array}$ \\
\hline 99RN502 & 395453 & 6911241 & Iron stained, light gray, pyritic, hornfelsed, melafelsic (quartz-feldspar-bearing), laminated volcanic(?) rock. \\
\hline 99RN526C & 394507 & 6916120 & Chalcopyrite + quart + malachite vein in amygdaloidal melabasalumetagabbro(?). \\
\hline 99JS29J & 395241 & 6915063 & Float of brick red to orange weathering limestone. \\
\hline 99JS41A & 390062 & 6918720 & White weathering quartz blocks ( 40 to $60 \mathrm{~cm}$ across) with limonite staining on fractures. \\
\hline 99JS46C & 393641 & 6913728 & Azurite-rich fracture coatings (up to $4 \mathrm{~mm}$ thick) in amygdaloidal lava. \\
\hline $995566 \mathrm{D}$ & 390485 & 6908246 & 1-mm-wide quartz veins with limonite staining. \\
\hline 99MBW549A & 390968 & 6919881 & $\begin{array}{l}\text { Milky quartz vein ( } 1 \text { foot wide) in amygdaloidal basalt with epidole + chlorite }+/ \text { - trace earthy hematite(?) + } \\
\text { malachite. }\end{array}$ \\
\hline 99MBW523 & 392087 & 6908650 & Pyrite altered, white and black, medium grained homblende dionte veined by quartz and pyrite. \\
\hline $99 M B N 436$ & 394865 & 6914901 & $\begin{array}{l}\text { Iron-stained pyritic zone approximately } 50 \text { foot wide crossing ridgeline. A green foliated metavolcantc rock is } \\
\text { mixed in with rusty rubble. }\end{array}$ \\
\hline 99ARJ033 & 393657 & 6915147 & Massive basalt with trace disseminated chalcopyrile(?) or pyrite(?). \\
\hline 99ARJ041 & $3 \overline{93814}$ & 6922343 & Gossan zone near conlact between metabasalt and metasedimentary rocks. \\
\hline 99ARJ056 & 392643 & 6924159 & $\begin{array}{l}\text { Limonite stained gossanous zone in metasedimentary rock with dark maroonish-gray fine grained layers } \\
\text { (dominate component) and thin pale gray coarser grained layers. }\end{array}$ \\
\hline 99PE011A & 392826 & 6913543 & Dark grayish-green, very line grained greenstone with disseminated pyrite. \\
\hline 99RN511A & 390274 & 6919764 & Iron stained, dark green, chlorite schist(?) cut by thin (up to $1.5 \mathrm{~cm}$ wide) quartz + pyrite + chalcopyrite(?) veins \\
\hline 99JS28B & 393528 & 6912149 & Very fractured, pyrite-bearing cherty rock with gray and cream-colored streaks and laminations. \\
\hline $99.5929 \mathrm{~K}$ & 395195 & 6915073 & Quartz veined metamafic schist with pyrite and iron oxides. \\
\hline $99 \mathrm{JS35C}$ & 393316 & 6921529 & Dark red-weathering zone in melamorphosed microgabbro. \\
\hline $99 J S 41 D$ & 389625 & 6918689 & Epidote altered, +1- magnetite-rich amygdaloidal basalt with minor malachite staining. \\
\hline $99 J 546 \mathrm{D}$ & 393641 & 6913728 & Orange weathering, green colored, chlorite-rich, schistose amygdaloidal lava with malachite staining. \\
\hline 99MBW525 & 392147 & 6908791 & $\begin{array}{l}\text { Black and white, medium grained, equigranular to porphyritic (huge quartz phenocrysts), biotite quartz diorite } \\
\text { with disseminated pyrite alteration, that is cut by numerous quartz + pyrite veins. }\end{array}$ \\
\hline 99 MBW559 & 396793 & 6919281 & $\begin{array}{l}\text { Two old prospecting trenches; one is } \sim 50 \text { feet by } 20 \text { feet, and one is }-10 \text { feet by } 10 \text { feet. Amygdaloidal basalt } \\
\text { with fractures coated with azurite, chalcocite, bornite, hematite, and (or) minor epidote. }\end{array}$ \\
\hline 99MBW547A & 390915 & 6919791 & Massive milky quartz + pyrite vein. \\
\hline $99 \mathrm{MBW} 441 \mathrm{~B}$ & 392555 & 6919849 & Quartz + limonite veins cutting porphyritic dacite dike. \\
\hline 99ARJI034A & 393096 & 6915371 & White quartz + hematite +1 - epidote veins in greenstone. \\
\hline
\end{tabular}


Table 1. Location and description of rock samples collected in the Iron Creek area for trace element gecochemical analyses.

\begin{tabular}{|c|c|c|c|}
\hline Sample & UTME & UTMN & Description \\
\hline 99ARJ042B & 393563 & 6922591 & Thin vein in altered granitic pluton. \\
\hline 99ARJ052B & 392105 & 6925027 & Slightly altered and pyritized, fine grained mafic intrusive or flow. \\
\hline 99PE11B & 392826 & 6913543 & Quartz + specular hemalite + limonite patches + crystalline and massive malachite veins in greenstone. \\
\hline 99RN511B & 390274 & 6919764 & $\begin{array}{l}\text { Iron stained, dark green chlorite schis(l) cut by chalcopyrite and quartz veins (up to } 1 \mathrm{~cm} \text { wide) with malachite } \\
\text { coatings. }\end{array}$ \\
\hline 99JS28F & 393528 & 6912149 & Orange weathering area in mudstone with quartz pebbles. \\
\hline 99JS31D & 392972 & 6919502 & Red weathering goethite materlal from a 1- to 2-cm-thick calcite vein. \\
\hline 99JS35D & 393261 & 6921533 & $\begin{array}{l}\text { Gossanous outcrop of medium gray to greenish gray, fine grained, granular silica rock with } 2-25 \% \text { fine to } \\
\text { medlum grained pyrite. }\end{array}$ \\
\hline 99JS44A & 396595 & 6913527 & Pyrrhotite-rich ( $10-15 \%)$, dark green, fine grained gabbro with local iron oxide coatings. \\
\hline 99JS46E & 393641 & 6913728 & Maroon to green colored amygdaloidal lava with epidote alteration and rare malachite coatings. \\
\hline 99MBW529B & 396749 & 6915574 & Very iron-stained, pyritic, felsic melavolcanic rock with minor quartz veining. \\
\hline 99MBW565 & 393954 & 6907704 & Small, 10 foot wide, iron-stalned, $+/$ - quartz veined zone trending -190 degrees. \\
\hline 99MBW405B & 381335 & 6920333 & $\begin{array}{l}\text { Disseminated magnetite, pyrite, and chalcopyrite in a medium green aphanitic rock cut by thin quartz }+ \text { limonite } \\
\text { veins. }\end{array}$ \\
\hline 99MBW442 & 392541 & 6919909 & Dark green finely crystalline gabbro cut by dacite dikes and quartz + pyrite veins. \\
\hline 99ARJ034C & 393096 & 6915371 & Hematite veinlet in very epldote and chlorite altered microgabbro. \\
\hline 99ARJ046 & 395544 & 6919678 & $\begin{array}{l}\text { Gossan(?) in mottled pale pink and pale green, quartz-rich, slightly porphyritic (quartz phenos up to } 4 \text { mm) } \\
\text { granite. }\end{array}$ \\
\hline 99ARJ073B & 385084 & 6911230 & $\begin{array}{l}\text { Dark green, equigranular, very fine grained greenstone with numerous quartz + epidote }+/ \text { - pyrite veins and } \\
\text { disseminated epidote. }\end{array}$ \\
\hline 99PE20A & 393265 & 6919290 & Green metamafic rock that is +1 - highly silicified, with areas of quartz + pyrite $+i$ - epidote in veins(?). \\
\hline 99RN517 & 389255 & 6910810 & Medium gray, pyritic meta-argillite. \\
\hline 99.JS28K & 393404 & 6912406 & $\begin{array}{l}\text { White colored siliceous rock with orange weathering limonite which may be alteration or a different original } \\
\text { jlthology(?). }\end{array}$ \\
\hline 99JS32日 & 392417 & 6919883 & $\begin{array}{l}\text { Orange weathering materlal (with patches of dark red, dark orange, and brown) with manganese(?) staining. } \\
\text { which contains very fine grained pyrite, that occurs near a dark green, hornfelsed metagabbro(?). }\end{array}$ \\
\hline 99.JS35E & 393261 & 6921533 & $\begin{array}{l}1-3 \mathrm{~mm} \text { thick, dark brown to black, manganese oxide crust on a gossanous outcrop of medium gray to } \\
\text { greenish gray, fine grained, granular silica rock with } 2-25 \% \text { fine to medium grained pyrite. }\end{array}$ \\
\hline 99JS44B & 396595 & 6913527 & Orange and yellow weathering, platey muscovite-quartz schist. \\
\hline 99JS50 & 395661 & 6912428 & $\begin{array}{l}\text { Orange-stained, light greenish-gray, cream, and gray layers in a porphyritic aphanite (flow or tuff) with }-2-4 \% \\
\text { blue-gray quartz phenocrysts and } 1-2 \% \text { fine grained disseminated pyrite. }\end{array}$ \\
\hline
\end{tabular}


Table 1. Location and description of rock samples collected in the Iron Creek area for trace element geochemical analyses.

\begin{tabular}{|c|c|c|c|}
\hline Sample & UTME & UTMN & Description \\
\hline 99MBW530 & 396123 & 6915607 & White, siliceous, quartz veined, quartz-sericite schist. \\
\hline 99MBW567 & 393850 & 6907893 & $\begin{array}{l}\text { Bright orange, iron stained zone approximately } 50 \text { feet wide with abundant fine grained disseminated pyrite in } \\
\text { gray siticeous rock. }\end{array}$ \\
\hline $99 M B W 417$ & 385096 & 6910383 & $\begin{array}{l}\text { Medium green, very fine grained, granular metagabbro/metavolcanic(?) rock cut by } 1 " \text { crystalline quartz veins } \\
\text { with areas of iron staining where pyrite is altering to limonite }\end{array}$ \\
\hline $99 \mathrm{MBW} 449 \mathrm{~B}$ & 392719 & 6919758 & $\begin{array}{l}\text { 30-foot-wide porphyritic dike that is iron stained and cut by quartz + pyrite + chalcopyrite(?) + limonite veins up } \\
\text { to } 1 \text { foot thick, which occur in a sub-parallel set. }\end{array}$ \\
\hline 99ARJ037A & $\overline{393534}$ & 6920040 & Iron-stained gossan zone ( 3 meters wide) with abundant red hematite. \\
\hline 99ARJ0718 & 393084 & 6908569 & $\begin{array}{l}\text { Gossanous iron-stained zone in schistose, vaguely bedded(?), metaluffs/lavas(?) that are intensely fractured } \\
\text { and faulted. Soft gouge occurs in fautted areas. }\end{array}$ \\
\hline 99PE009A & 392634 & 6913648 & $\begin{array}{l}\text { Massive magnetite, hematite, limonite and quartz occur in a linear, reddish-stained zone within dark greenish- } \\
\text { gray, aphanitic, amygdaloidal greenstone. Much of the iron mineral-bearing material is weathered out to } \\
\text { boxworks. }\end{array}$ \\
\hline 99PE53 & 390282 & $6 \overline{9} 19745$ & Smail, $\sim 1$-foot-thick, rusty stained zones with sulfides in greenstone. \\
\hline 99RN524A & 394673 & 6916185 & Very epidote altered metamafic rock cut by chalcedony(?) + pyrite + chalcopyrite(?) veins and masses. \\
\hline 99.JS29A & 395648 & 6914721 & $\begin{array}{l}\text { Gray to light yeilow-orange weathering, white mica quartz schist with }-2 \% \text { very fine grained disseminated } \\
\text { pyrite. }\end{array}$ \\
\hline 99.JS33B & 393629 & $6 \overline{921975}$ & Bright orange weathering cakcite veins in metabasalt. \\
\hline 99JS39D & 394350 & 6921333 & $\begin{array}{l}\text { Orange weathering, calcareous + quartz material with orange to red-brown boxworks, but with no obvious } \\
\text { sulfides. }\end{array}$ \\
\hline $99 \mathrm{~J} \$ 45 \mathrm{~A}$ & 395642 & 6913155 & $\begin{array}{l}\text { Pale green, fine grained, equigranular, altered, quartz-feldspar dacite(?) with up to } 15 \% \text { disseminated pyrite } \\
\text { (grains }<1 \mathrm{~mm} \text { in diameter). }\end{array}$ \\
\hline 99JS57A & 383559 & 6909858 & $\begin{array}{l}\text { Small, } 2 \text {-meter-wide, orange-siained zone of bright, light and medium green, hornfelsed, metamafic(?) rocks } \\
\text { cut by veins of quartz + red hematite, and calcite (or iron carbonate). }\end{array}$ \\
\hline 99MBW535 & 395919 & 6915744 & 2-foot-wide fault with inlense pyrite alteration adjacent to very foliated dark green chlorite phyllite. \\
\hline 99MBW513 & 395263 & 6913394 & Fault zone with intensely calcite- and iron carbonale-altered/veined, tan weathering, foliated rock. \\
\hline $99 \mathrm{MBW} 420 \mathrm{~A}$ & 395835 & 6914617 & $\begin{array}{l}\text { Epidote altered and veined greenstone cut by sparse veins of quartz + epidote + limonite (after sulfides(?)) + } \\
\text { carbonate(?) + possible tremolite(?) (or wetl-developed slicks? in quarz). }\end{array}$ \\
\hline $99 \mathrm{MBW} 449 \mathrm{C}$ & 392719 & 6919758 & Malachite and azurite in sheared metagabbro float derived from rocks coming down from eastern hillside. \\
\hline 99MBW408A & 380674 & 6920509 & Hornfelsed volcaniclastic conglomerate/breccia with finely disseminated pyrite. \\
\hline 99MBW544 & 390683 & 6919566 & $\begin{array}{l}\text { Trachyte dike with color-zoned, pink-gray white, square-shaped feldspar phenocrysts, that is cut by } 1 \text { - to } 2-\mathrm{mm} \text { - } \\
\text { wide, pale gray quartz veins with } 1 \mathrm{~mm} \text { by } 1 \mathrm{~mm} \text { limonite patches (pseudomorphs after sulfide). }\end{array}$ \\
\hline
\end{tabular}


Table 1. Location and description of rock samples collected in the Iron Creek area for trace element geochemical analyses.

\begin{tabular}{|c|c|c|c|}
\hline Sample & UTME & UTMN & Description \\
\hline 99ARj044B & 393146 & 6923847 & $\begin{array}{l}\text { Light tan and gray, angular limestone fragments (up to } 1 \text { inch) cemented by a calcareous/clay(?) matrix. A 1- } \\
\text { to } 2 \text {-foot-wide hand dug trench is found within a hematile-stained area. }\end{array}$ \\
\hline $83 \mathrm{Hn} 83 \mathrm{a}$ & 392870 & 6913760 & $\begin{array}{l}\text { 30-foot-wide zone of chlorite-altered greenstone cut by quartz + hematite }+/ \text { - pyrite }+/ \text { - chalcopyrite }(+/- \\
\text { pyrrhotite(?) or perhaps magnetite(?)) veins. }\end{array}$ \\
\hline $83 \mathrm{H} n 83 \mathrm{~b}$ & 392870 & 6913760 & $\begin{array}{l}\text { 30-foot-wide zone of chlorite-altered greenslone cul by quartz + hernatite }+/ \text { - pyrite }+/ \text { chalcopyrite }(+/- \\
\text { pyrrhotite(?) or perhaps magnetite(?)) veins. }\end{array}$ \\
\hline $83 \mathrm{Hn} 83 \mathrm{c}$ & 392870 & 6913760 & $\begin{array}{l}\text { 30-foot-wide zone of chlonite-altered greenstone cut by quart + hernatile }+/ \text { - pyrite }+/ \text { - chalcopyrite }(+/- \\
\text { pyrrhotite(?) or perhaps magnetite(?)) veins. }\end{array}$ \\
\hline $83 \mathrm{Hn} 84$ & 393060 & 6913510 & $\begin{array}{l}\text { Possible sphalerite(?) occurs as very finely disseminated pink/brown colored patches in quartzose rock with } \\
\text { abundant pyrite. }\end{array}$ \\
\hline $83 \mathrm{Hn} 107$ & 394110 & 6913770 & $\begin{array}{l}\text { Medium green, aphanitic, amygdaloidal greenstone that is faulted and chlonte altered. Rock is cut by vein of } \\
\text { quartz + hematite, and it is copper stained. }\end{array}$ \\
\hline $83 \mathrm{Hnt} 08$ & 394130 & 6913790 & $\begin{array}{l}\text { Greenstone with pods and small, randomly-oriented veinlets of quartz + hematite + pyrite + chalcopyrite + } \\
\text { copper oxide. }\end{array}$ \\
\hline $83 H n 128$ & 392840 & 6914100 & Greenstone with pods of pyrite + arsenopyrite(?) + hematite + quartz. \\
\hline $83 \mathrm{Hn} 134$ & 393460 & 6912100 & Mixed andesite(?) and a pinkish-green, fine grained, massive zone that is siliceous(?) and plag-rich. \\
\hline $83 \mathrm{Hn}+36$ & 393275 & 6912030 & $\begin{array}{l}\text { Grenstone with numerous iron-stained gossans with pyrite }+ \text { chalcopyrite(?) patches and within small shears } \\
\text { (minor). }\end{array}$ \\
\hline $83 \mathrm{H} \cap 139$ & 394940 & 6913530 & $\begin{array}{l}\text { Light green to purplish zone in altered intermediate volcanic(?) rock with quartz crystals, which contains } 3-5 \% \\
\text { pyrite (disseminated and in small veinlets). }\end{array}$ \\
\hline $83 \mathrm{Hn} 146$ & 393410 & 6912500 & Rusly-weathering mineralization at the contact between limestone and tuff. \\
\hline $83 \mathrm{Hn} 166$ & 395900 & 6911090 & $\begin{array}{l}\text { Fine grained to aphanitic, chlorite altered, slightly foliated, intermediate(?) volcanic rock with minor pale blue } \\
\text { quartz phenocrysts, that is cut by epidote-filled fractures. }\end{array}$ \\
\hline $83 \mathrm{Hn} 168$ & 395650 & 6911190 & $\begin{array}{l}\text { Orange-stained, bedded(?) horizon of siliceous rock composed of blue-gray, massive quartz with } \\
\text { disseminations and veinlets of pyrite and sphalerite(?) ( } \sim 5 \% \text { combined sulfides). }\end{array}$ \\
\hline $83 \mathrm{Hn} 170$ & 395530 & 6911230 & Slightly foliated/bedded(?) siliceous rock with chlorite(?), blue quartz eyes, and disseminated pyrite. \\
\hline $83 \mathrm{Hn} 175$ & 394730 & 6911200 & Altered granodiorite with quartz + pyrite + chlorite alteration. \\
\hline $83 \mathrm{H} n 177$ & 394920 & 6911300 & Veinlets in metamorphosed crystal tuff(?). \\
\hline $83 \mathrm{Hn} 178$ & 394990 & 6911450 & Folded quartz veins in greenschist(?). \\
\hline $83 \mathrm{Hn} 184$ & 393040 & 6912510 & Tuff - andesite contact. \\
\hline $83 \mathrm{Hn} 192.2$ & 392800 & 6913600 & Quartz + hematite + pyrite + chalcopyrite(?)-bearing rock. \\
\hline
\end{tabular}


Table 1. Location and description of rock samples collected in the Iron Creek area for trace element geochemical analyses.

\begin{tabular}{|c|c|c|c|}
\hline Sample & UTM E & UTMN & Description \\
\hline $83 \mathrm{Hn} 192.3$ & 392800 & 6913600 & $\begin{array}{l}\text { Quartz and hematite in crude layers (mm to } \mathrm{cm} \text { scale), with relative quartz:hematite percentages varying from } \\
\text { 50:50 to almost } 300 \% \text { hematite. }\end{array}$ \\
\hline $83 \mathrm{Hn} 192.4$ & 392800 & 6913600 & $\begin{array}{l}\text { Dark green, chlorite-altered andesite(?) cut by quartz }+1 \text { - hematite stringers from } 3 \mathrm{~mm} \text { to } 2.5 \mathrm{~cm} \text { that both } \\
\text { cross-cut and are paralel to bedding. Up to } 1 \% \text { pyrite and (or) chalcopyrite in some veins. }\end{array}$ \\
\hline $83 \mathrm{Hn} 192.5$ & 392800 & 6913600 & $\begin{array}{l}\text { Dark green, chlorite altered, magnetic andesite(?) with quartz (up to } 15 \%)+ \text { hematite (5-10\%) + pyrite } \\
\text { occurring in pods and veins. }\end{array}$ \\
\hline $83 \mathrm{Hn} 192.6$ & 392800 & 6913600 & $\begin{array}{l}\text { Chlorite altered andesite(?) with quartz, hematite, and sulfides (up to } 3 \% \text { ) that are locally abundant and } \\
\text { randomly distributed. }\end{array}$ \\
\hline $83 \mathrm{Hn}\{92.7$ & 392800 & 6913600 & Iron stained, altered andesite(?) with quartz + sulfides (locally up to $5 \%$ ) + minor hematite throughout rock. \\
\hline $83 \mathrm{Hn} 192.8$ & 392800 & 6913600 & Slightly iron stained, allered andesite with quartz + hematite + sulfide $(2-3 \%)$ veins. \\
\hline $83 \mathrm{Hn} 192.9$ & 392800 & 6913600 & Massive hematite, quart, and pyrite ( $\mathrm{i}-2 \%)$ that appears to grade laterally into andesite(?). \\
\hline
\end{tabular}


Table 2. Concentration of trace clements in rock samples collected in the Iron Creek area.

Note: na = not anaiyzed; Intf = interference between the spectral lines of $C u$ with those of Bi and $P$ in high Cu samples

\begin{tabular}{|c|c|c|c|c|c|c|c|c|c|c|c|c|c|c|c|c|c|c|c|c|c|}
\hline SAMPLE & $\mathrm{Au}$ & $\mathrm{Ag}$ & $\mathrm{Ag}$ & Al & As & $\mathrm{B}$ & Ba & $\mathrm{Be}$ & $B \mathrm{~B}$ & Bi" & $\mathrm{Ca}$ & $\mathrm{Cd}$ & $\mathrm{Co}$ & $\mathrm{Cr}$ & $\mathrm{Cu}$ & $\mathrm{Cu}$ & $\mathrm{Fe}$ & $\mathrm{Ga}$ & $\mathrm{Ge}$ & $\mathrm{Hg}$ & $\mathrm{Hg}$ \\
\hline & $\mathrm{ppb}$ & $\mathrm{ppm}$ & opt & $\%$ & $\mathrm{ppm}$ & ppm & $\mathrm{ppm}$ & $\mathrm{ppm}$ & ppm & $\mathrm{ppm}$ & $\%$ & $\mathrm{ppm}$ & ppm & $\mathrm{ppm}$ & $\mathrm{ppm}$ & $\%$ & $\%$ & ppm & ppm & $\mathrm{ppm}$ & ppb \\
\hline 99ARJ029 & 50 & 0.6 & na & 3.33 & 14 & $<10$ & 10 & $<0.5$ & $<2$ & na & 0.52 & $<0.5$ & 45 & 198 & 772 & na & 8.88 & $<10$ & na & 3 & $<10$ \\
\hline 99ARJ038A & 10 & 0.4 & na & 2.04 & 8 & $<10$ & 100 & $<0.5$ & $<2$ & na & 0.12 & $<0.5$ & 18 & 86 & 229 & $n a$ & 4.86 & $<10$ & na & $\overline{3}$ & 10 \\
\hline 99ARJ069 & 25 & $<0.2$ & na & 3.59 & 10 & $<10$ & 10 & $<0.5$ & $<2$ & na & 3.66 & $<0.5$ & 44 & 122 & 175 & na & 6.75 & 10 & na & $<1$ & na \\
\hline 9QPEOI0A & 205 & 4.6 & na & 0.78 & 146 & $<10$ & 410 & $<0.5$ & Intf & 1.6 & 0.7 & $<0.5$ & 24 & 165 & $>10000$ & 2.16 & $>15.00$ & $<10$ & na & $<1$ & na \\
\hline 99PE63 & 65 & 13.6 & na & 0.55 & 284 & $<10$ & 10 & $<0.5$ & 10 & ne & 0.01 & 1.5 & 148 & 214 & 3780 & $\mathrm{na}$ & 8.91 & $<10$ & na & 4 & 4780 \\
\hline $99 \mathrm{RN} 525$ & 5 & $<0.2$ & na & $\uparrow .64$ & $<2$ & $<10$ & 20 & $<0.5$ & 48 & na & $0 . \overline{59}$ & $<0.5$ & 22 & 85 & 35 & na & $>15.00$ & $<10$ & na & 3 & 80 \\
\hline 99J\$29C & 10 & 0.4 & กа & 5.15 & 2 & $<10$ & 100 & $<0.5$ & $<2$ & na & 2.57 & $<0.5$ & 33 & 49 & 146 & na & 4.92 & 10 & na & $<1$ & na \\
\hline $99 . J 533 \mathrm{C}$ & $<5$ & 0.8 & па & 3.48 & 8 & $<10$ & 70 & $<0.5$ & $<2$ & na & 2.69 & $<0.5$ & 12 & 27 & 246 & na & 11.95 & $<10$ & $n a$ & 2 & na \\
\hline $99 J S 40 \mathrm{~B}$ & $<5$ & $<0.2$ & na & 1.55 & 90 & $<10$ & 120 & $<0.5$ & $<2$ & na & 1.06 & 40.5 & 14 & 64 & 57 & na & 2.19 & $<10$ & na & $<1$ & na \\
\hline $99 \mathrm{JS} 46 \mathrm{~A}$ & 65 & 11 & na & 3.43 & $<2$ & $<10$ & 70 & $<0.5$ & Intf & na & 0.98 & $<0.5$ & 25 & 86 & $>10000$ & 5.84 & 6.58 & 10 & na & $<1$ & na \\
\hline 99J558A & $<5$ & $<0.2$ & na & 4.1 & $<2$ & $<10$ & 90 & $<0.5$ & $<2$ & na & 0.64 & $<0.5$ & 28 & 106 & 292 & na & 12.45 & $<10$ & na & 1 & 20 \\
\hline 99MBW537 & 15 & 1.4 & na & 0.96 & 20 & $<10$ & 40 & $<0.5$ & $<2$ & na & 0.02 & $<0.5$ & 69 & 131 & 1025 & na & $>15.00$ & $<10$ & na & 2 & 520 \\
\hline 99MBW515B & $<5$ & 0.8 & na & 0.33 & $<2$ & $<10$ & 40 & $<0.5$ & $<2$ & na & 0.1 & 40.5 & 2 & 207 & 17 & na & $0 . \overline{73}$ & $<10$ & na & $<1$ & $n a$ \\
\hline $99 \mathrm{MB} W 430 \mathrm{~B}$ & 25 & 9.6 & na & 0.52 & 178 & $<10$ & 30 & $<0.5$ & 8 & па & 0.36 & $<0.5$ & 490 & 147 & 8650 & na & 9.59 & $<10$ & $\mathrm{na}$ & 1 & 290 \\
\hline $99 \mathrm{MBW} 515$ & 45 & 1.2 & na & 2.8 & 2 & $<10$ & 140 & $<0.5$ & $<2$ & na & 0.55 & $<0.5$ & 12 & 105 & 81 & na & 6.18 & $<10$ & $n a$ & 1 & $<10$ \\
\hline 99ARJO30B & $<5$ & $<0.2$ & กa & 0.43 & 12 & $<10$ & 10 & $<0.5$ & $<2$ & na & 7.19 & $<0.5$ & 4 & 150 & 21 & na & 0.86 & $<10$ & na & $<1$ & $\mathrm{na}$ \\
\hline 99AR.J038B & 30 & 0.8 & na & 1.4 & 20 & $<10$ & 10 & $<0.5$ & 2 & na & 0.03 & $<0.5$ & 12 & 196 & 596 & na & 3.53 & $<10$ & na & $<1$ & na \\
\hline 99ARJ068 & $<5$ & $<0.2$ & na & 2.5 & $<2$ & $<10$ & 40 & $<0.5$ & $<2$ & na & 1.25 & $<0.5$ & 30 & 119 & 20 & na & 3.95 & $<10$ & na & $<1$ & na \\
\hline 99PE010B & 140 & 3.6 & na & 2.48 & $\leq 2$ & $<10$ & 390 & $<0.5$ & 16 & na & 0.07 & $<0.5$ & 28 & 88 & 8110 & na & 9.44 & $<10$ & na & $<1$ & na \\
\hline $99 R N 502$ & 40 & 1.6 & na & 3.28 & 18 & $<10$ & 70 & $<0.5$ & $<2$ & na & 0.09 & 6.5 & 13 & 39 & 121 & na & 4.93 & $<10$ & na & 1 & 340 \\
\hline 99RN526C & $<5$ & 0.2 & na & 1.71 & $<2$ & $<10$ & 200 & $<0.5$ & 8 & na & 1.12 & $<0.5$ & 18 & 109 & 6780 & $\mathrm{na}$ & 3.91 & $<10$ & na & $<1$ & na \\
\hline $99 J 529 \mathrm{~J}$ & $<5$ & 0.2 & na & 1.57 & 30 & $<10$ & 40 & $<0.5$ & $<2$ & na & 11.9 & $<0.5$ & 16 & 51 & 31 & na & $2 . \overline{99}$ & $<10$ & па & $<1$ & $n a$ \\
\hline 99JS41A & $<5$ & $<0.2$ & na & 0.21 & $<2$ & $<10$ & $<10$ & $<0.5$ & $<2$ & na & 0.05 & $<0.5$ & 3 & 393 & 17 & $\mathrm{na}$ & 0.8 & $<10$ & na & $<1$ & na \\
\hline $99 . \mathrm{S} 46 \mathrm{C}$ & 100 & 10.6 & na & 3.19 & $<2$ & $<10$ & 10 & $<0.5$ & $\ln t f$ & na & 0.84 & $<0.5$ & 28 & 82 & $>10000$ & 4.06 & 6.89 & 10 & na & $<1$ & na \\
\hline 99 JS660 & 10 & 0.2 & na & 0.51 & 6 & $<10$ & 10 & $<0.5$ & $<2$ & na & 0.07 & $<0.5$ & 1 & 92 & 99 & $\mathrm{na}$ & 1.77 & $<10$ & na & $<1$ & na \\
\hline $99 \mathrm{MBW} 549 \mathrm{~A}$ & 270 & 6.2 & na & 0.42 & 2 & $<10$ & $\angle 10$ & $<0.5$ & 6 & na & 0.18 & $<0.5$ & 16 & 197 & 5260 & $\pi a$ & 2.91 & $<10$ & na & $<1$ & na \\
\hline 99MBW523 & $<5$ & $<0.2$ & na & 1.78 & 6 & $<10$ & 160 & $<0.5$ & $<2$ & na & 0.67 & $<0.5$ & 3 & 111 & 136 & na & 1.65 & $<10$ & na & $<1$ & $\mathrm{na}$ \\
\hline 99MBW436 & $<5$ & 2.6 & па & 1 & 184 & $<10$ & 10 & $<0.5$ & 2 & na & 0.07 & $<0.5$ & 206 & 119 & 964 & na & $>15.00$ & $<10$ & na & 1 & 310 \\
\hline 99ARJ033 & $<5$ & $<0.2$ & na & 4.59 & $<2$ & $<10$ & 110 & $<0.5$ & $<2$ & na & 0.47 & $<0.5$ & 51 & 194 & 1140 & na & 7.69 & 10 & na & $<1$ & na \\
\hline 99ARJ041 & $<5$ & 0.2 & na & 2.39 & $\overline{100}$ & $<10$ & 20 & $<0.5$ & $<2$ & na & $0 . \dot{81}$ & $<0.5$ & 92 & 476 & 581 & na & 4.83 & $<10$ & na & 1 & na \\
\hline 99ARJo56 & $<5$ & $<0.2$ & na & 2.91 & 2 & $<10$ & 60 & $<0.5$ & $<2$ & na & 0.52 & $<0.5$ & 19 & 87 & 142 & na & 4.46 & $<10$ & na & $<1$ & na \\
\hline 99PE011A & 10 & $<0.2$ & na & 5.11 & $<2$ & $<10$ & 10 & $<0.5$ & $<2$ & na & 0.14 & $<0.5$ & 26 & 84 & 419 & na & 9.52 & 10 & na & $<1$ & па \\
\hline 99RN511A & 210 & $>100.0$ & 3.5 & 5.65 & 18 & $<10$ & $<10$ & $<0.5$ & Intf & $\mathrm{ma}$ & 0.01 & 1 & 335 & 178 & $>10000$ & 5.87 & $>15.00$ & 20 & na & $<1$ & na \\
\hline $99 \mathrm{JS} 28 \mathrm{~B}$ & $<5$ & 0.2 & na & 0.29 & 14 & $<10$ & $4 \overline{0}$ & $<0.5$ & $<2$ & па & 0.16 & $<0.5$ & 1 & 79 & \begin{tabular}{|l|}
97 \\
\end{tabular} & na & 0.6 & $<10$ & na & $<1$ & na \\
\hline
\end{tabular}


Table 2. Concentration of trace elements in rock samples collected in the lron Creek area.

Note: $n a=$ not analyzed: intf $=$ interference between the spectral lines of $C u$ with those of $B j$ and $P$ in high $C u$ samples.

\begin{tabular}{|c|c|c|c|c|c|c|c|c|c|c|c|c|c|c|c|c|c|c|c|c|c|}
\hline SAMPLE & $\mathrm{Au}$ & $\mathrm{Ag}$ & $\mathrm{Ag}$ & $\mathrm{Al}$ & $\overline{\mathrm{As}}$ & $\overline{8}$ & $\mathrm{Ba}$ & $\mathrm{Be}$ & $\bar{B} i$ & $B i^{m+}$ & $\mathrm{Ca}$ & $\mathrm{cd}$ & $\mathrm{Co}$ & $\mathrm{Cr}$ & $\mathrm{Cu}$ & $\mathrm{Cu}$ & $\mathrm{Fe}_{0}$ & $\mathrm{Ga}$ & $\mathrm{Ge}$ & $\mathrm{Hg}$ & $\mathrm{Hg}^{\prime \prime}$ \\
\hline & $p \rho b$ & $\mathrm{ppm}$ & opt & $\%$ & $\mathrm{ppm}$ & $\mathrm{ppm}$ & ppm & $\mathrm{ppm}$ & $\mathrm{ppm}$ & ppm & $\%$ & $\mathrm{ppm}$ & $\mathrm{ppm}$ & $\mathrm{ppm}$ & $\mathrm{ppm}$ & $\%$ & $\%$ & ppm & $\mathrm{ppm}$ & $\mathrm{ppm}$ & $\rho p b$ \\
\hline 99JS29K & 35 & 6.2 & na & 1.02 & 46 & $<10$ & 20 & $<0.5$ & 8 & na & 0.01 & $<0.5$ & 91 & 177 & 9070 & na & 6.18 & $<10$ & na & 1 & 330 \\
\hline 99.JS35C & $<5$ & $<0.2$ & na & 2.14 & $<2$ & $<10$ & 10 & $<0.5$ & $<2$ & na & 1.48 & $<0.5$ & 23 & 25 & 153 & $\mathrm{na}$ & 4.34 & $<10$ & na & $<1$ & na \\
\hline 99JS41D & 5 & 1.8 & na & 1.2 & 8 & $<10$ & $<10$ & $<0.5$ & 6 & na & 0.05 & $<0.5$ & 62 & 308 & 2980 & na & 4.27 & $<10$ & na & 1 & 80 \\
\hline 99JS46D & 150 & 15.2 & na & 2.39 & $<2$ & $<10$ & 10 & $<0.5$ & Intf & na & 0.85 & $<0.5$ & 13 & 70 & $>10000$ & 5.49 & 4.29 & $<10$ & na & $<1$ & na \\
\hline $99 \mathrm{MBW} 525$ & $<5$ & 0.2 & na & 1.21 & 12 & $<10$ & 110 & $<0.5$ & $<2$ & na & 0.29 & $<0.5$ & 6 & 112 & 274 & na & 2.56 & $<10$ & na & $<1$ & na \\
\hline 99MBW559 & 270 & $>100.0$ & 3.8 & 1.76 & $<2$ & $<10$ & 10 & $<0.5$ & Intf & na & 0.06 & $<0.5$ & $<1$ & 6 & $>10000$ & 31.5 & 7.81 & $<10$ & กล & $<1$ & na \\
\hline 99MBW547A & $<5$ & 1.8 & na & 0.04 & $<2$ & $<10$ & $<10$ & $<0.5$ & $<2$ & na & 0.02 & $<0.5$ & 2 & 297 & 3480 & na & 1.09 & $<10$ & na & $<1$ & na \\
\hline 99றMB̈W441B & $<5$ & $<0.2$ & na & 0.47 & $<2$ & $<10$ & 90 & $<0.5$ & $<2$ & na & 0.16 & $<0.5$ & 2 & 227 & 358 & na & 0.81 & $<10$ & na & $<1$ & na \\
\hline 99ARJ034Ä & $<5$ & $<0.2$ & na & 0.49 & $<2$ & $<10$ & $<10$ & $<0.5$ & $<2$ & na & $<.01$ & 40.5 & 9 & 121 & 102 & na & 14.75 & $<10$ & na & 1 & 20 \\
\hline 99ARJ042B & $<5$ & $<0.2$ & $\mathrm{na}$ & 0.44 & $<2$ & $<10$ & $<10$ & $<0.5$ & $<2$ & na & 0.07 & $<0.5$ & $<1$ & 163 & 136 & na & 0.64 & $<10$ & na & $<1$ & na \\
\hline 99ARJ0528 & $<5$ & $<0.2$ & na & 2.29 & $<2$ & $<10$ & 190 & $<0.5$ & $<2$ & na & 2.83 & $<0.5$ & $\overline{13}$ & 34 & 58 & na & 4.13 & $<10$ & na & $<1$ & na \\
\hline 99PE11B & 15 & 12.6 & na & 0.18 & $<2$ & $<10$ & $<10$ & $<0.5$ & Intf & na & $<.01$ & $<0.5$ & $<1$ & 117 & $>10000$ & 4.12 & 13.15 & $<10$ & กอ & $<1$ & na \\
\hline 99RN5118 & 205 & 22.6 & na & 5.08 & $\overline{124}$ & $<10$ & $<10$ & $<0.5$ & $\ln t f$ & 1.7 & 0.05 & 2 & 376 & 109 & $>10000$ & 3.71 & $>15.00$ & 10 & na & $<1$ & na \\
\hline $99 . \mathrm{S} 28 \mathrm{~F}$ & $<5$ & 0.2 & na & 0.49 & 14 & $<10$ & 40 & $<0.5$ & $<2$ & na & 5.03 & $<0.5$ & 11 & 49 & 380 & na & 3.59 & $<10$ & na & 1 & 10 \\
\hline 99JS31D & 15 & $<0.2$ & na & 0.33 & $<2$ & $<10$ & 10 & $<0.5$ & $<2$ & na & 12.35 & $<0.5$ & 29 & 27 & 207 & na & 4.71 & $<10$ & na & $<1$ & na \\
\hline 99.JS35D & 10 & $<0.2$ & $n a$ & 2.12 & 44 & $<10$ & 40 & $<0.5$ & $<2$ & na & 0.65 & 0.5 & 5 & 52 & 147 & na & 3.91 & $<10$ & na & $<1$ & na \\
\hline 99.JS44A & $<5$ & 0.2 & $\mathrm{na}$ & 2.68 & $<2$ & $<10$ & 130 & $<0.5$ & $<2$ & na & 1.02 & $<0.5$ & 17 & 32 & 75 & na & 5.61 & $<10$ & па & $<1$ & na \\
\hline 99JS46E & $<5$ & $<0.2$ & na & 3.52 & $<2$ & $<10$ & 50 & $<0.5$ & 6 & na & 1.52 & $<0.5$ & 32 & 93 & 9530 & na & 6.42 & 10 & na & $<1$ & na \\
\hline $99 \mathrm{MBW} 52 \overline{9 B}$ & 70 & 2.4 & na & 0.42 & 312 & $<10$ & 150 & $<0.5$ & $<2$ & na & 0.02 & $<0.5$ & $<1$ & 70 & 47 & na & 2.36 & $<10$ & na & 2 & 1500 \\
\hline 99MBW565 & $<5$ & $<0.2$ & na & 0.71 & $<2$ & $<10$ & 20 & $<0.5$ & $<2$ & na & 0.05 & $<0.5$ & 1 & 184 & 93 & na & 1.97 & $<10$ & na & $<1$ & na \\
\hline $99 \mathrm{MBW} 405 \mathrm{~B}$ & 95 & 0.8 & na & 5.35 & $<2$ & $<10$ & 30 & $<0.5$ & $<2$ & $n a$ & 3.77 & $<\overline{0,5}$ & 30 & 139 & 903 & na & 2.96 & 10 & na & $<1$ & na \\
\hline $99 \mathrm{MBW} 442$ & $<5$ & 0.2 & na & 0.71 & 4 & $<10$ & 10 & $<0.5$ & 52 & na & 0.37 & $<0.5$ & 34 & 237 & 401 & na & 5.27 & $<10$ & na & 1 & 10 \\
\hline $99 \mathrm{AR} J 034 \mathrm{C}$ & $<5$ & $<0.2$ & па & 3.88 & $<2$ & $<10$ & 10 & 80.5 & $<2$ & na & 1.33 & $<0.5$ & 40 & 100 & 128 & na & 6.27 & $<10$ & na & $<1$ & na \\
\hline 99ARJ046 & $<5$ & $<0.2$ & na & 0.54 & $<2$ & $<10$ & 90 & $<0.5$ & $<2$ & na & 0.07 & $<0.5$ & 1 & 158 & 31 & na & 0.88 & $<10$ & na & $<1$ & $\mathrm{na}$ \\
\hline 99ARJ073B & $<5$ & $<0.2$ & na & 2.32 & $<2$ & $<10$ & 60 & $<0.5$ & $<2$ & na & 1.13 & $<0.5$ & 27 & 124 & 46 & na & 4.04 & $<10$ & na & $<1$ & na \\
\hline 99PE20A & $<5$ & $<0.2$ & na & 1.18 & $<2$ & $<10$ & $<10$ & $<0.5$ & $<2$ & $\mathrm{na}$ & 1.56 & $<0.5$ & 36 & 120 & 61 & na & 2.68 & $\leq 10$ & na & 1 & $<10$ \\
\hline 99RN517 & $<5$ & 0.2 & na & 1.95 & 6 & $<10$ & 30 & $<0.5$ & $<2$ & $n a$ & 1.23 & 1 & 5 & 97 & 42 & na & 2.47 & $<10$ & na & $<1$ & na \\
\hline 99JS28K & 5 & $<0.2$ & na & 2.08 & 2 & $<10$ & 20 & $<0.5$ & $<2$ & na & 2.16 & $<0.5$ & 3 & 217 & 35 & na & 1.45 & $<10$ & na & 1 & $<10$ \\
\hline $99 \mathrm{~J} 332 \mathrm{~B}$ & $<5$ & $<0.2$ & na & 1.49 & 8 & $<10$ & 10 & $<0.5$ & $<2$ & na & 1.61 & $<0.5$ & 17 & 64 & 96 & na & 4.25 & $<10$ & na & $<1$ & na \\
\hline 99JS35E & 10 & $<0.2$ & na & 2.42 & 108 & $<10$ & 40 & $<0.5$ & $<2$ & na & 1.63 & $<0.5$ & $\overline{4}$ & 127 & 145 & na & 4.52 & $<10$ & na & $<1$ & na \\
\hline 99.JS44B & $<5$ & 0.2 & na & 1.41 & $<2$ & $<10$ & 50 & 0.5 & $<2$ & na & 0.23 & $<0.5$ & 9 & 111 & 39 & na & 3.4 & $<10$ & na & $<1$ & na \\
\hline 99JS50 & $<5$ & $<0.2$ & $\mathrm{na}$ & 0.85 & 12 & $<10$ & 80 & $<0.5$ & $<2$ & na & 0.1 & $<0.5$ & 2 & 88 & 7 & na & 1.84 & $<10$ & na & $<1$ & na \\
\hline $99 \mathrm{MB}=530$ & 15 & 1.2 & na & 0.29 & 66 & $<10$ & 640 & 40.5 & $<2$ & na & 4.01 & $<0 . \overline{5}$ & $<1$ & 191 & 38 & na & 0.73 & $<10$ & na & 6 & 6560 \\
\hline 99MBW567 & 10 & $<0.2$ & ra & $2.6 \overline{3}$ & $<2$ & $<10$ & 30 & $<0.5$ & $<2$ & na & 0.24 & $<0.5$ & $1 \overline{3}$ & 85 & 24 & na & 5.52 & $<10$ & na & 1 & 20 \\
\hline
\end{tabular}




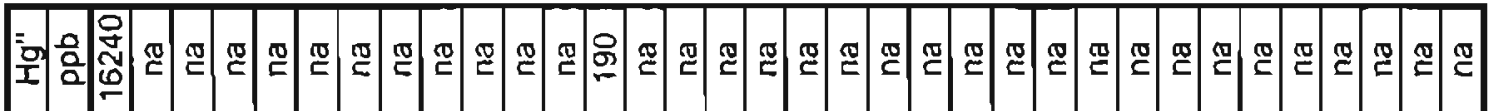

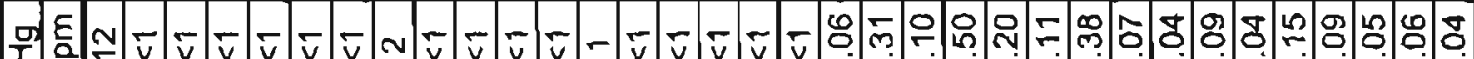

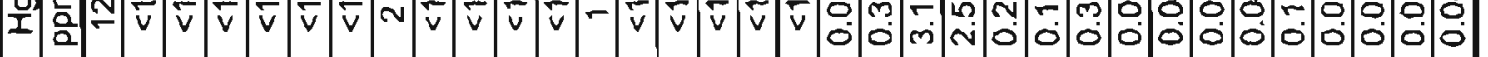

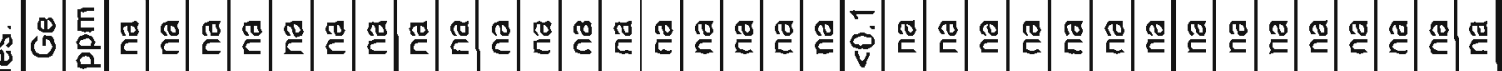

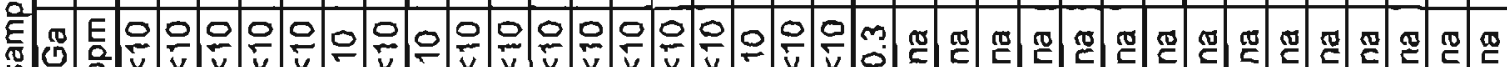

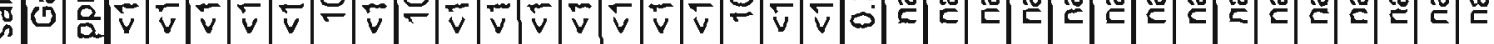

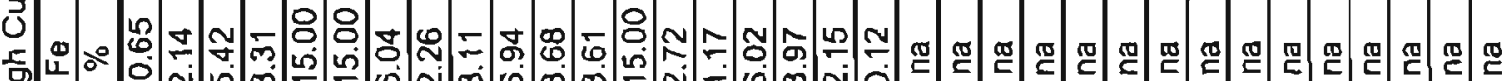
䔶

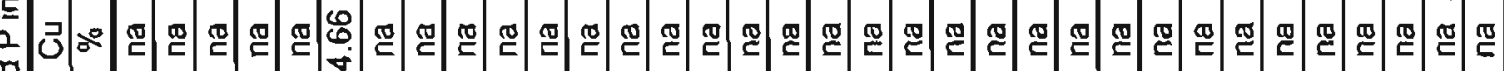
JE

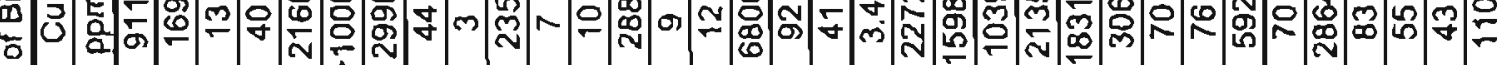

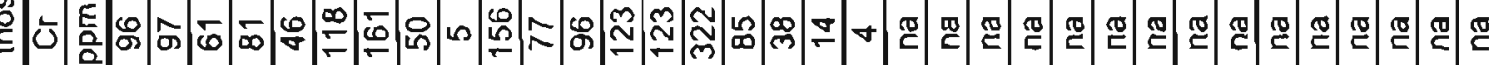

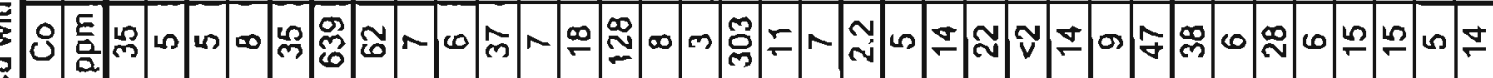

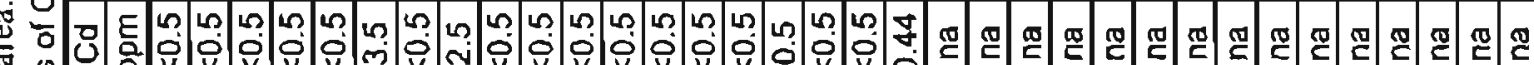
要要

o 制

을

$\stackrel{2}{x}$

巨

要

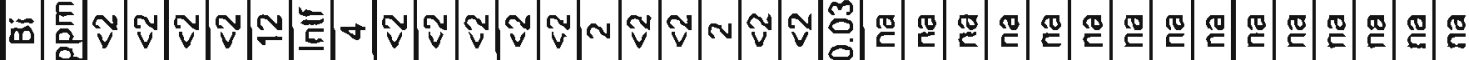

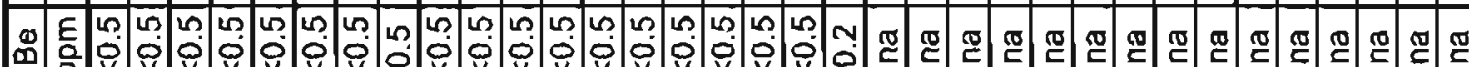

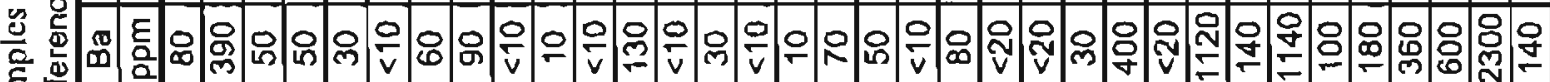
赵 क E

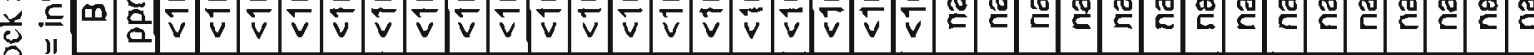

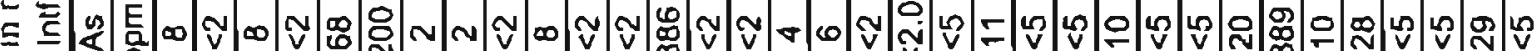

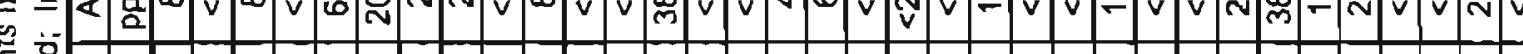

8

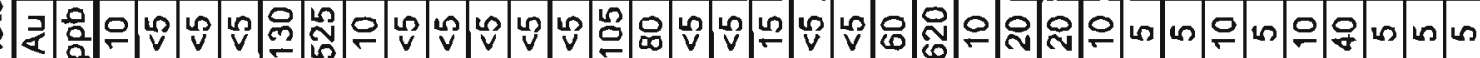
导

\&

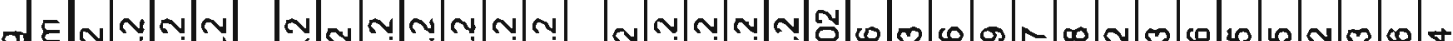
\&

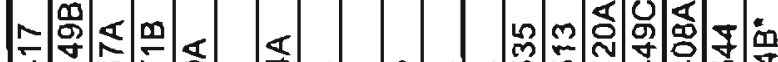

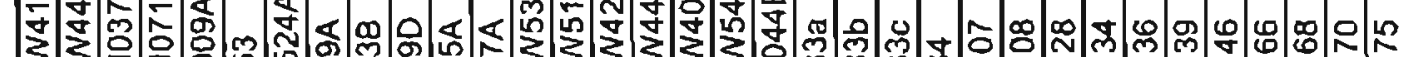

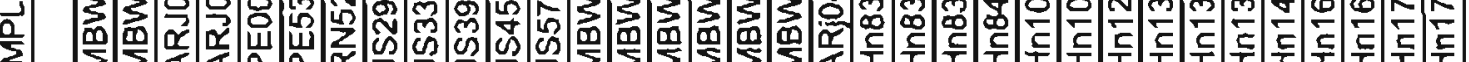




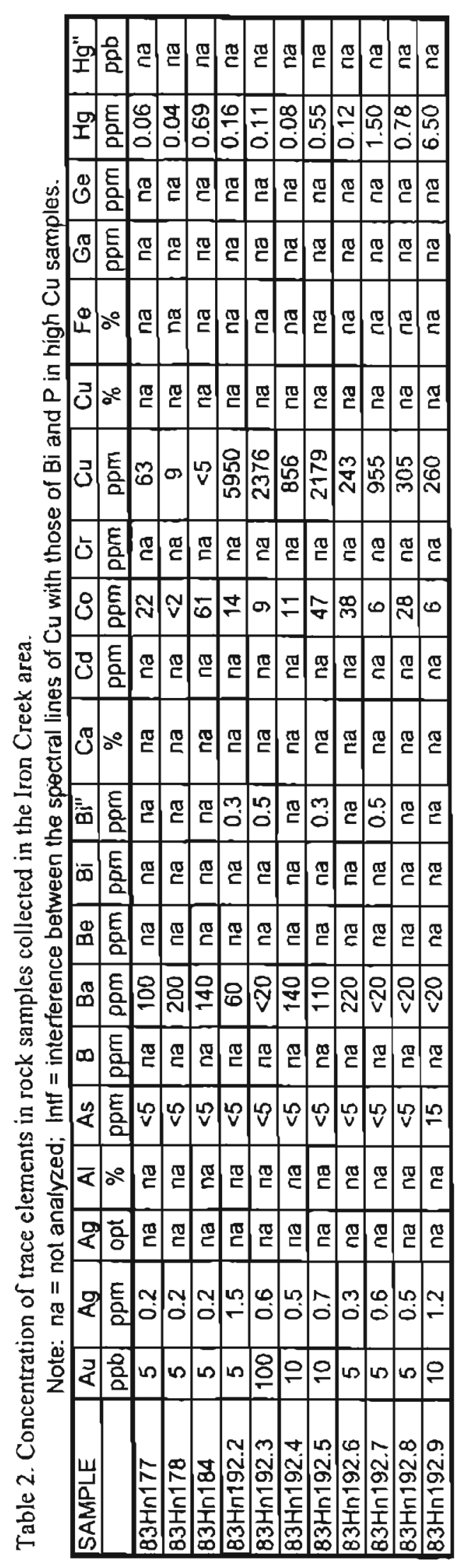


\& N

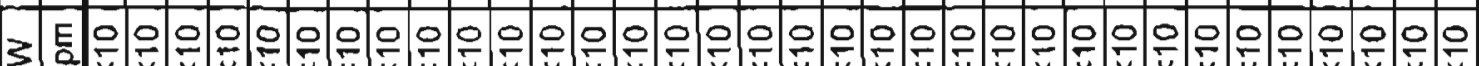

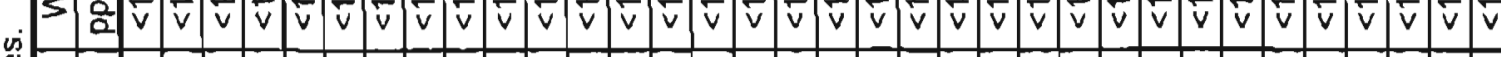

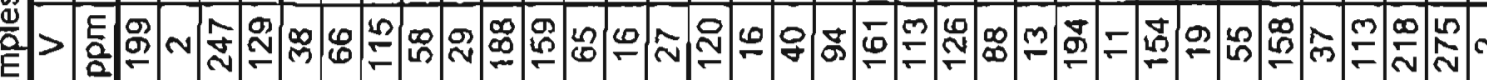
出

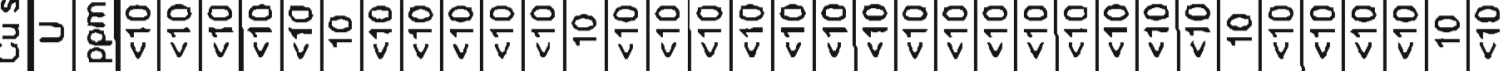

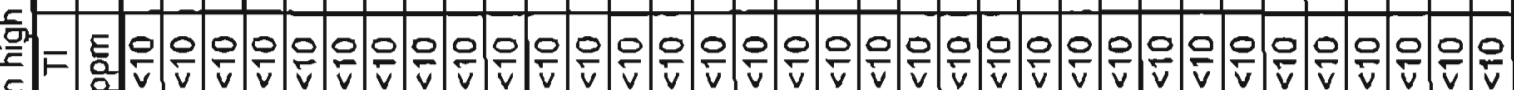

DE

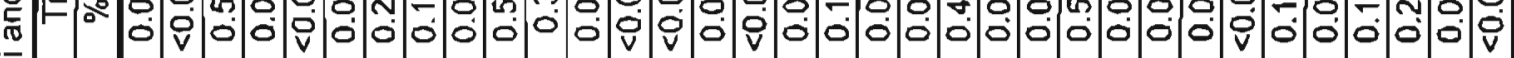

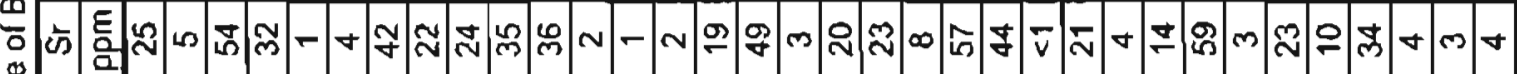

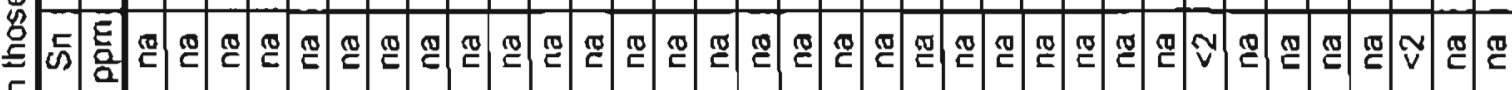

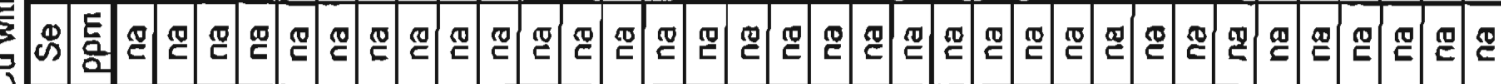

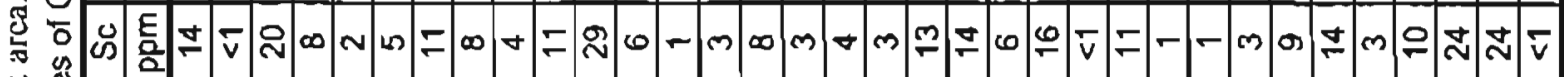

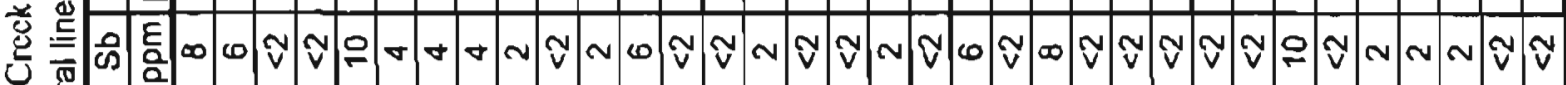

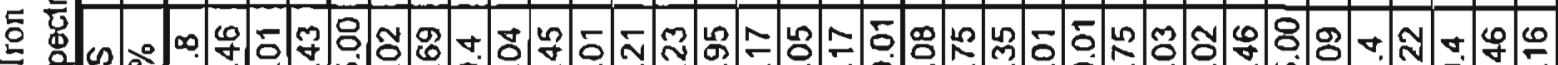

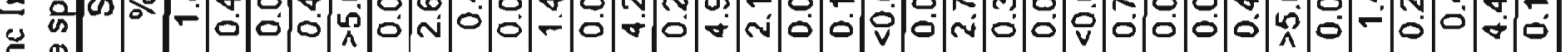
至

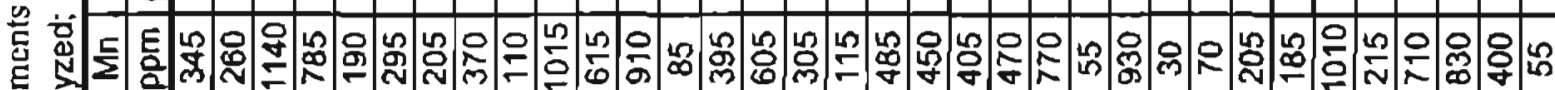

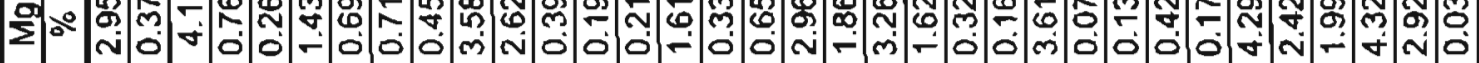

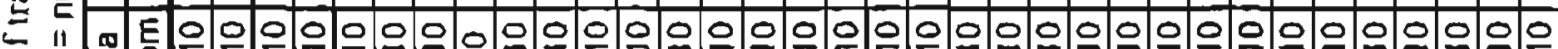

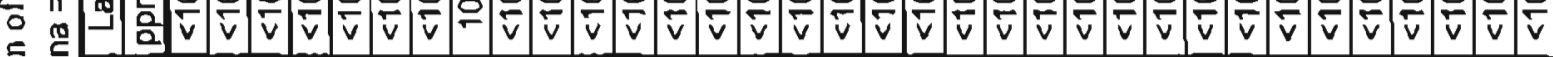
을 O. 
Table 2. Concentration of trace elements in rock samples collected in the lron Creck area.

Note: na $=$ not analyzed; Intf $=$ interference between the spectral lines of $\mathrm{Cu}$ with those of $\mathrm{Bi}$ and $\mathrm{P}$ in high $\mathrm{Cu}$ samples.

\begin{tabular}{|c|c|c|c|c|c|c|c|c|c|c|c|c|c|c|c|c|c|c|c|c|c|c|}
\hline SAMPLE & $\mathrm{K}$ & La & $\mathrm{Mg}$ & $\mathrm{Mn}$ & Mo & $\mathrm{Na}$ & $\mathrm{Ni}$ & $P$ & $\mathrm{~Pb}$ & $\bar{s}$ & Sb & $\mathrm{Sc}$ & Se & $\mathrm{Sn}$ & $\mathrm{Sr}$ & $\mathrm{Ti}$ & $\mathrm{TI}$ & $\mathrm{U}$ & $\mathrm{V}$ & W & Zn & Te \\
\hline & $\%$ & $\mathrm{ppm}$ & $\%$ & ppm & ppm & $\%$ & ppm & $\rho \rho m$ & $p p m$ & $\%$ & ppm & $\mathrm{ppm}$ & $\mathrm{ppm}$ & $\mathrm{ppm}$ & ppn & $\%$ & ppm & $\mathrm{ppm}$ & $\mathrm{ppm}$ & ppm & $\mathrm{ppm}$ & \\
\hline $99 \mathrm{JS} 29 \mathrm{~K}$ & 0.06 & $<10$ & 0.33 & 170 & 10 & $<0.01$ & 25 & 90 & $<2$ & 1.53 & $<2$ & 3 & $\mathrm{na}$ & na & 1 & $<0.01$ & $<10$ & $<10$ & 36 & $<10$ & 22 & na \\
\hline 99JS35C & 0.05 & $<10$ & $0 . \overline{76}$ & 330 & $<1$ & 0.08 & 6 & 720 & 2 & 0.37 & $<2$ & 8 & na & na & 14 & 0.23 & $<10$ & $\angle 10$ & 136 & $<10$ & 40 & na \\
\hline 99.JS41D & $<0.01$ & $<10$ & $\overline{0.73}$ & 300 & $<1$ & $<0.01$ & 20 & 40 & 2 & $<0.01$ & $<2$ & 3 & $\mathrm{na}$ & $<2$ & 2 & 0.02 & $\angle 10$ & $<10$ & 64 & $<10$ & 104 & na \\
\hline$\overline{99 J S 46 D}$ & $<0.01$ & $<10$ & 2.71 & 620 & $<1$ & 0.04 & $\overline{32}$ & Intf & $<2$ & $\{.14$ & $<2$ & 5 & na & na & 23 & 0.53 & $<10$ & $<10$ & 141 & $<10$ & 100 & na \\
\hline 99MBW525 & 0.42 & $<10$ & 0.39 & 275 & $\overline{44}$ & 0.12 & 4 & 40 & $<2$ & 1.1 & $<2$ & 1 & na & $<2$ & 68 & 0.05 & $<10$ & $\angle 10$ & 25 & $<10$ & 48 & na \\
\hline $99 M B W 559$ & $<0.01$ & $<10$ & 1,54 & $36 \overline{5}$ & $<1$ & $<0.01$ & $\overline{30}$ & Intf & $<2$ & $>5.00$ & $<2$ & 8 & na & na & 6 & 0.01 & 10 & $<10$ & 199 & $<10$ & 44 & na \\
\hline $99 \mathrm{MBW} 547 \mathrm{~A}$ & $<0.01$ & $<10$ & $\overline{0.01}$ & 25 & 3 & $<0.01$ & $\overline{4}$ & 10 & $<2$ & 0.66 & $<2$ & $\leq 1$ & na & $<2$ & $<1$ & $<0.01$ & $<10$ & $<10$ & 3 & $<10$ & $<2$ & na \\
\hline $99 \mathrm{MBW} 441 \mathrm{~B}$ & 0.07 & $<10$ & 0.14 & 120 & 15 & 0.05 & $\overline{3}$ & 120 & $<2$ & 0.04 & $<2$ & $<1$ & $n a$ & $<2$ & $\overline{29}$ & 0.01 & $<10$ & $<10$ & 17 & $<10$ & 16 & na \\
\hline 99ARJ034A & $<0.01$ & $<10$ & 0.27 & 85 & 8 & $<0.01$ & 9 & 10 & $<2$ & $<0.01$ & $<2$ & 1 & $\overline{n a}$ & $<2$ & 1 & 0.01 & $<10$ & $<10$ & 61 & 130 & 6 & na \\
\hline 99ARJ042B & 0.33 & $<10$ & $<.01$ & 150 & 1 & 0.12 & 1 & $<10$ & 4 & $<0.01$ & 2 & $c 1$ & na & $<2$ & $<1$ & $<0.01$ & $<10$ & $<10$ & $<1$ & $<10$ & 66 & na \\
\hline 99ARJ052B & 0.22 & $<10$ & 1.17 & 775 & 3 & 0.07 & $\overline{4}$ & 630 & 6 & 1.26 & $<2$ & 9 & na & $<2$ & 29 & 0.19 & $<10$ & $<10$ & 64 & 510 & 66 & na \\
\hline 99PE11B & $<0.01$ & $<10$ & 0.1 & 55 & $\overline{4}$ & $<0.01$ & 5 & In:f & 10 & 0.26 & $<2$ & 1 & na & na & 1 & $<0.01$ & $<10$ & $<10$ & 76 & 140 & 30 & $\overline{n a}$ \\
\hline 99RN511B & $<0.01$ & $<10$ & 2.65 & 400 & $<1$ & $<0.01$ & 79 & Intf & $\overline{c 2}$ & $>5.00$ & $<2$ & 20 & na & $<2$ & 1 & 0.06 & $<10$ & $<10$ & 220 & $<10$ & 472 & na \\
\hline 99JS28F & 0.15 & $<10$ & 0.73 & 930 & $<1$ & 0.01 & 29 & 270 & 8 & 0.12 & 2 & 3 & na & $<2$ & 152 & $<0.01$ & $<10$ & $<10$ & 15 & $<10$ & 52 & na \\
\hline 99 JS310 & 0.1 & $<10$ & 5.26 & $\overline{1095}$ & $<1$ & $<0.01$ & 73 & $<10$ & $<2$ & 0.05 & $<2$ & 5 & na & na & 162 & $<0.01$ & $<10$ & $<10$ & 45 & $<10$ & 38 & na \\
\hline 99.JS350 & 0.59 & $<10$ & 0.72 & 150 & $<1$ & 0.01 & 10 & 1240 & $<2$ & 0.32 & $<2$ & 7 & na & $\leq 2$ & 9 & 0.16 & $\angle 10$ & $<10$ & 67 & $\angle 10$ & 6 & na \\
\hline $99, \mathrm{JS} 4 \overline{\mathrm{A}}$ & 0.19 & $<10$ & 2.17 & 455 & $<1$ & 0.11 & 20 & 920 & 2 & 0.41 & 2 & 9 & na & na & 24 & 0.31 & $<10$ & $<10$ & 177 & $<10$ & 74 & na \\
\hline 99JS46E & $<0.01$ & $<10$ & 4.48 & 1275 & $<1$ & 0.04 & 63 & 490 & $<2$ & 0.02 & $<2$ & 11 & na & na & 6 & $\overline{0.61}$ & $<10$ & $<10$ & 208 & $<10$ & 140 & na \\
\hline $99 \mathrm{MBW} 529 \mathrm{~B}$ & 0.26 & $<10$ & 0.06 & 15 & 1 & $<0.01$ & 1 & 30 & 70 & 1.18 & 14 & $\leq 1$ & na & $<2$ & 4 & $<0.01$ & $<10$ & $<10$ & 3 & $<10$ & 8 & na \\
\hline 99MBW565 & 0.09 & $<10$ & 0.3 & 120 & 1 & 0.08 & $\overline{3}$ & 30 & $<2$ & 0.82 & 2 & 1 & $\mathrm{na}$ & $<2$ & 2 & $<0.01$ & $<10$ & $<10$ & 7 & $<10$ & 8 & na \\
\hline 99MBW405B & 0.05 & $<10$ & 0.71 & 225 & $<1$ & 0.5 & 26 & 680 & 2 & 0.1 & 2 & $\overline{9}$ & na & na & 181 & 0.26 & $<10$ & $<10$ & 144 & $<10$ & 24 & na \\
\hline $99 \mathrm{MBW} 442$ & $\overline{0.02}$ & $<10$ & 0.5 & 195 & 7 & 0.01 & 29 & 100 & $<2$ & 1.27 & $<2$ & $\overline{4}$ & na & $<2$ & 23 & 0.12 & $<10$ & $<10$ & 106 & $<10$ & 42 & กล \\
\hline 99ARJ034C & $<0.01$ & $<10$ & 3.58 & 835 & $<1$ & $\leq 0.01$ & 60 & 460 & 2 & $<0.01$ & $<2$ & 8 & na & na & 92 & 0.48 & $<1 \overline{0}$ & $<10$ & 139 & $<10$ & 66 & na \\
\hline 99ARJ046 & 0.34 & 30 & 0.05 & 80 & 1 & 0.14 & 3 & 10 & 2 & $<0.01$ & $<2$ & 1 & na & na & 6 & 0.01 & $<10$ & $<10$ & 4 & $<10$ & 6 & na \\
\hline 99ARJ073B & 0.32 & $<10$ & 2.08 & 515 & $<1$ & 0.1 & 53 & 450 & $<2$ & 0.03 & $<2$ & 5 & na & na & 22 & 0.42 & $<10$ & $<10$ & 126 & $<10$ & 26 & na \\
\hline 99PE20A & 0.01 & $<10$ & 0.33 & 95 & $<1$ & 0.02 & 35 & 200 & 2 & 1.12 & $<2$ & 4 & na & $<2$ & 87 & 0.25 & $<10$ & $<10$ & 71 & $<10$ & 2 & na \\
\hline 99RN517 & 0.13 & $<10$ & 0.22 & 175 & 9 & 0.26 & 21 & 1540 & 4 & 0.12 & 2 & 4 & na & $<2$ & 70 & 0.13 & $\leq 10$ & $<10$ & 66 & $<10$ & 90 & na \\
\hline $99 . J 528 \mathrm{~K}$ & 0.01 & $<10$ & $\overline{0.11}$ & 115 & $<1$ & $<0.01$ & 14 & 300 & 8 & 0.3 & $<2$ & 4 & na & $<2$ & 12 & 0.09 & $<10$ & $<10$ & 37 & $<10$ & 32 & na \\
\hline $99 \mathrm{JS} 32 \mathrm{~B}$ & 0.08 & $<10$ & 0.95 & 230 & $12 \uparrow$ & 0.09 & 33 & 690 & 8 & 0.88 & $<2$ & & na & $<2$ & 53 & 0.51 & $<10$ & $<10$ & 108 & $<10$ & 58 & na \\
\hline $99 \mathrm{JS} 35 \mathrm{E}$ & 0.53 & $<10$ & 0.36 & 150 & $<1$ & 0.02 & 7 & 350 & $<2$ & 0.34 & $<2$ & & na & na & 17 & 0.19 & $<10$ & $\leq 10$ & 82 & $\leq 10$ & 2 & na \\
\hline 99JS44B & 0.07 & $<10$ & 1.31 & 75 & 4 & 0.09 & 21 & 860 & 6 & 0.64 & $<2$ & 12 & na & $<2$ & 8 & $<0.01$ & $<10$ & $<10$ & 150 & $<10$ & 50 & na \\
\hline $99 . J 550$ & 0.06 & $<10$ & 0.49 & 100 & 9 & 0.12 & 2 & 230 & $<2$ & 0.44 & $<2$ & 7 & na & $<2$ & 6 & 0.01 & $\angle 10$ & $<10$ & 22 & $\angle 10$ & 40 & na \\
\hline $99 \mathrm{MBW} 530$ & 0.27 & 10 & $<.01$ & 5 & 3 & $<0.01$ & 2 & 10 & 132 & 0.06 & 26 & $<1$ & $\mathrm{na}$ & $<2$ & 5 & $<0.01$ & $\leq 10$ & $<10$ & 1 & $<10$ & 148 & na \\
\hline $99 M B W 567$ & 0.06 & $<10$ & 1.8 & 425 & $<1$ & 0.09 & 6 & 80 & 4 & 2.52 & 2 & 7 & na & $<2$ & 21 & $<0.01$ & $<10$ & $<10$ & 102 & $<10$ & 54 & na. \\
\hline
\end{tabular}




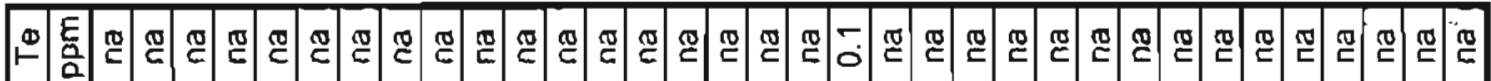

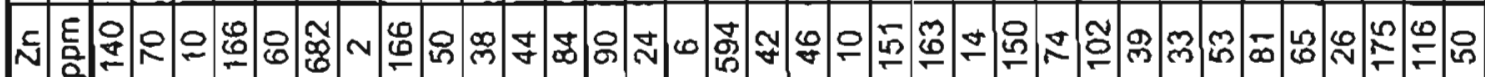

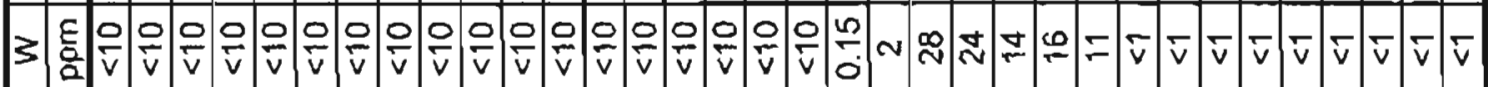

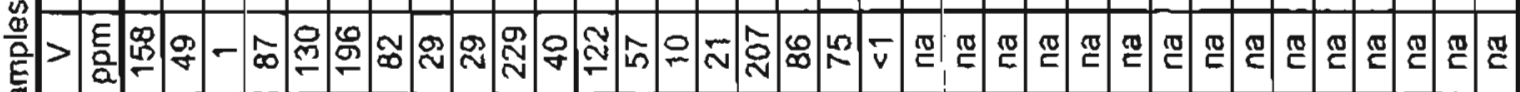

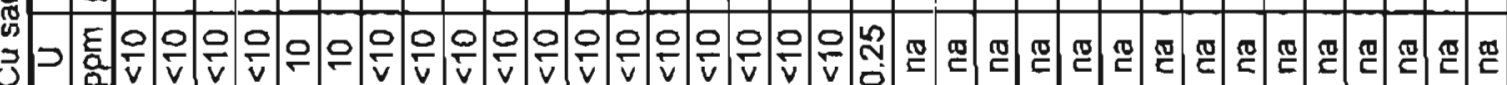

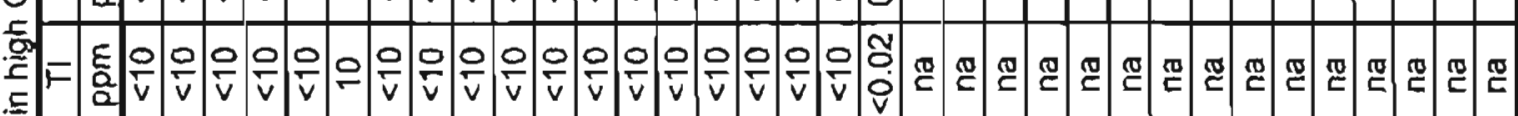

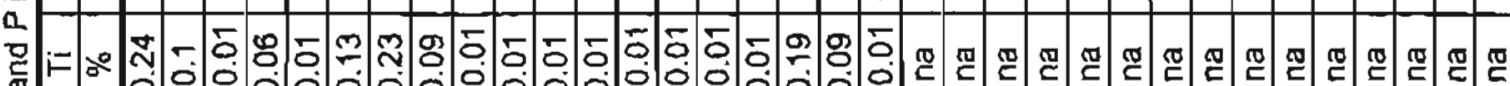

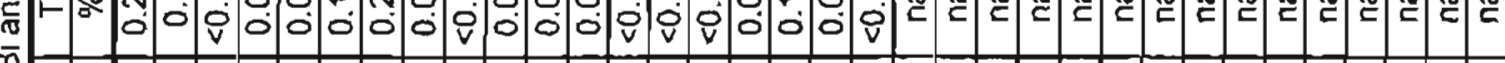

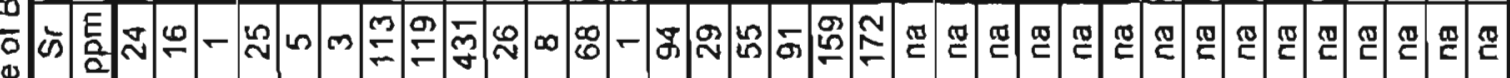

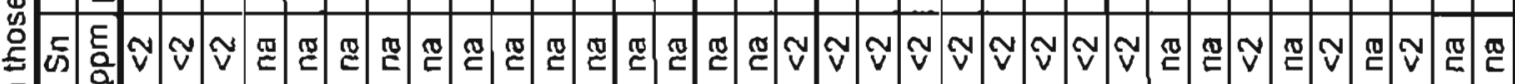

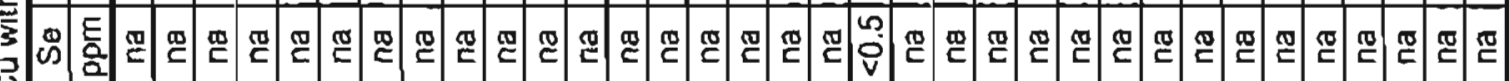

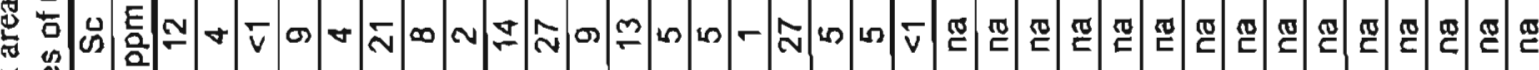

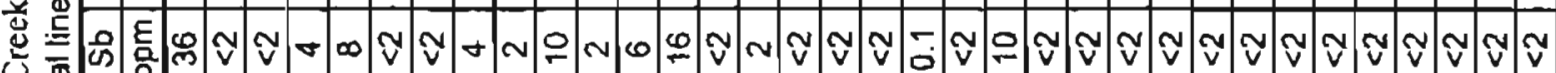

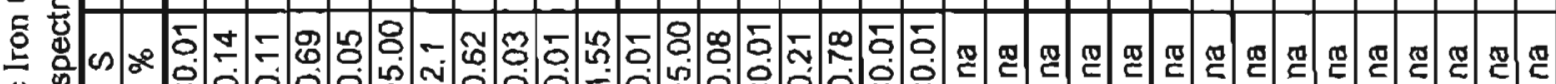
空

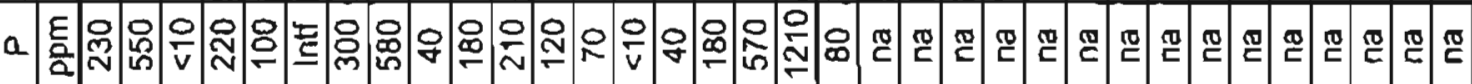

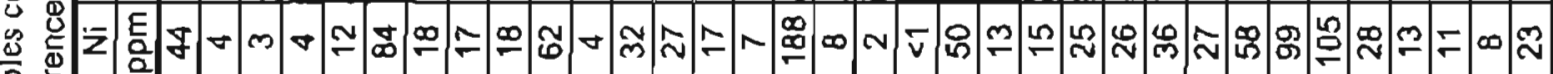

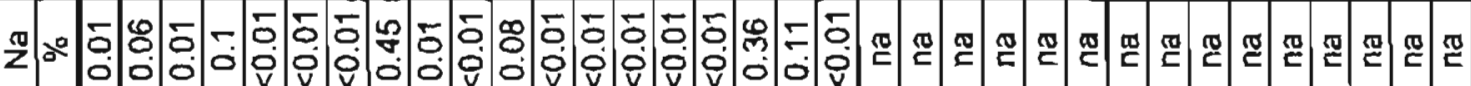

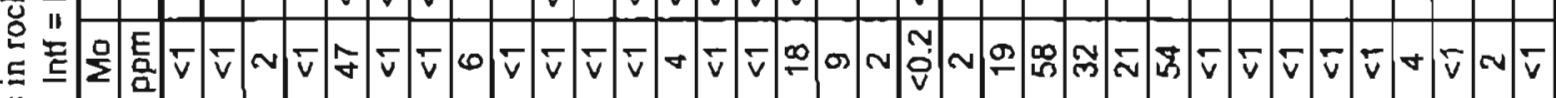
总 总 焉

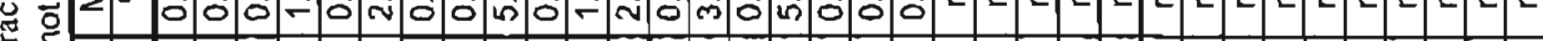

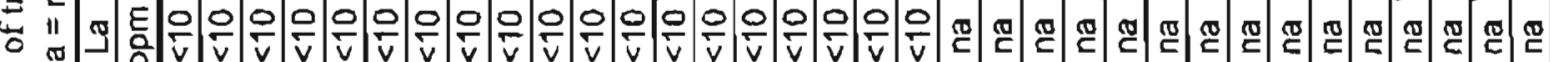




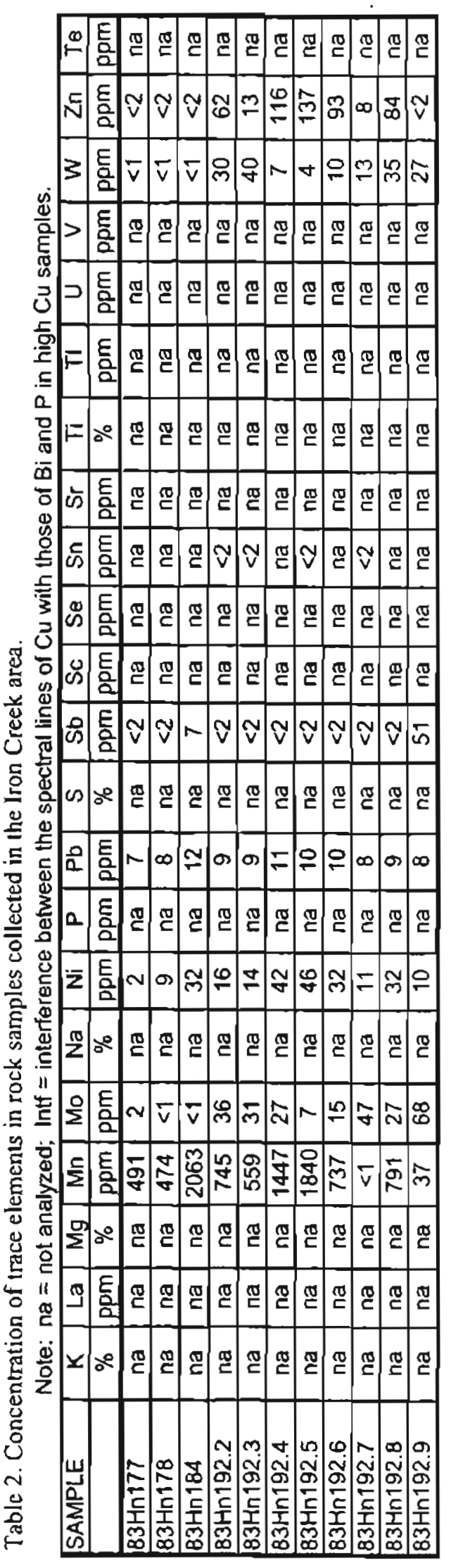


Table 3. Location and description of rock samples collected in the Iron Creek area for major-oxide, mitor oxide, and urace element analyses.

\begin{tabular}{|c|c|c|c|}
\hline Sample \# & UTHE & UTM N & Sample description \\
\hline 99ARjo27 & 382633 & 6908334 & 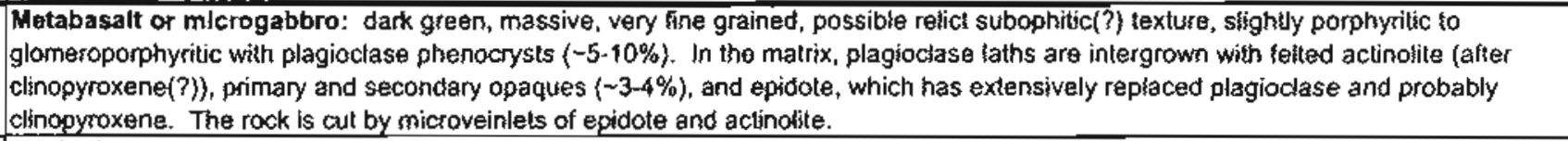 \\
\hline 99ARJ02B & 388127 & 6909105 & 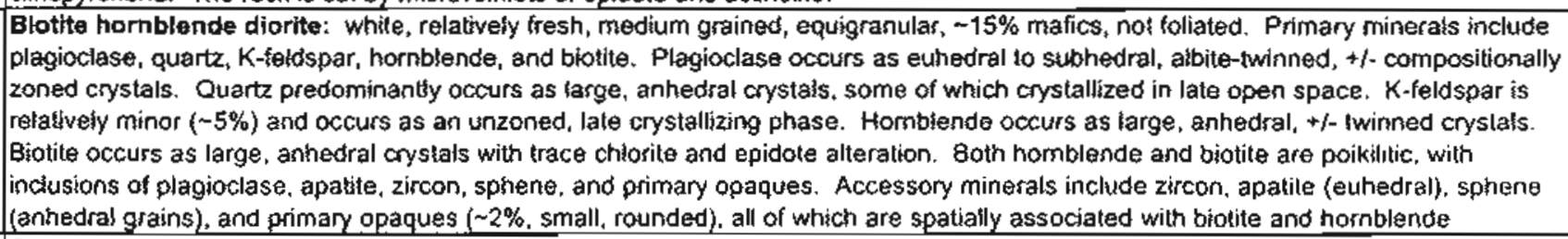 \\
\hline 99ARj032 & 394024 & 6912513 & 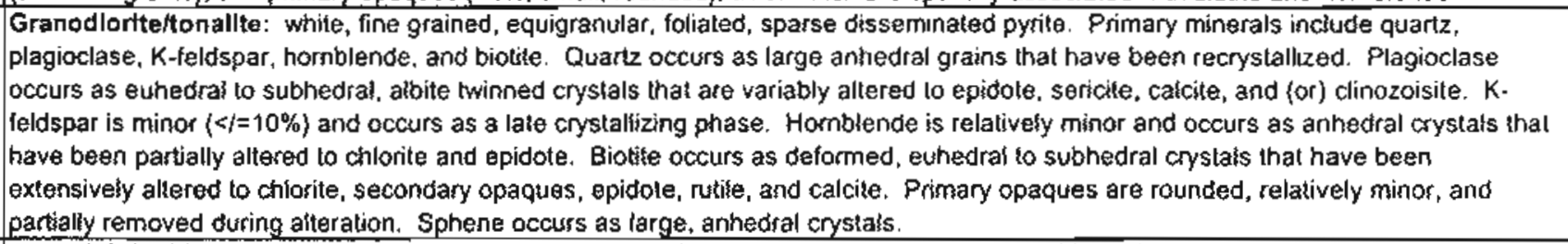 \\
\hline 99ARjO35B2 & 392615 & 6918663 & 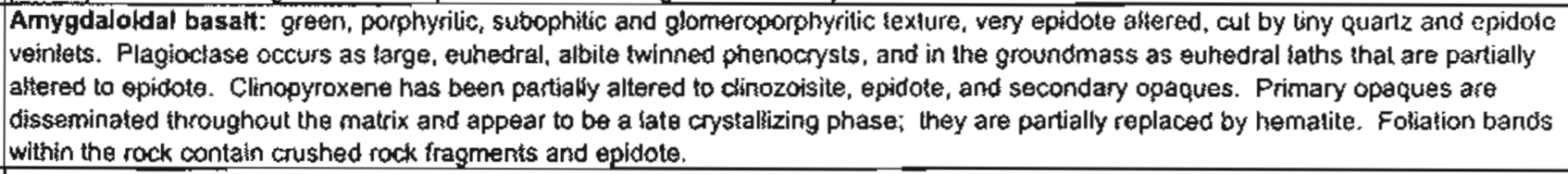 \\
\hline 99ARJO42A & 393557 & 6922594 & 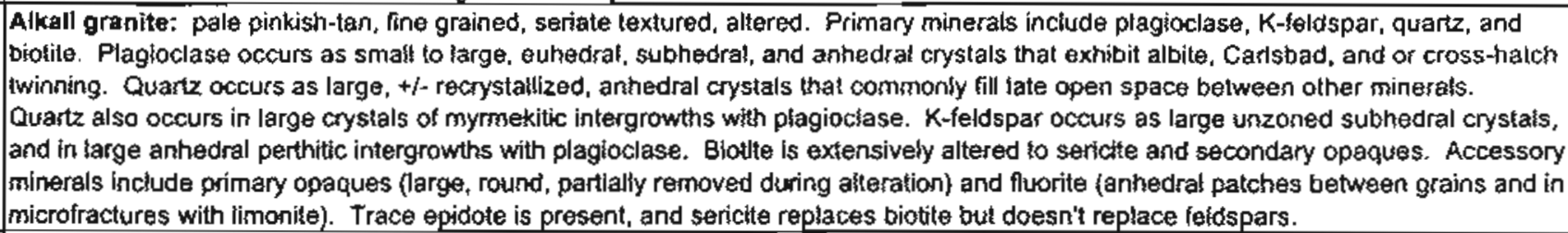 \\
\hline 99AR:068 & 394799 & 6917729 & $\begin{array}{l}\text { Amygdaloidal mlerogabbro: grayish green, amygdaloidal, epidole altered. Composed of } \sim 40 \% \text { clinopyroxene intergrown with }-50 \% \\
\text { plagloclase [euhedral, partially altered to sericite), }-5 \% \text { chlorite and epldote, and } 1 \% \text { primary, late-crystallizing opaques. }\end{array}$ \\
\hline 99ARjo72 & 392743 & $6907 \uparrow 26$ & 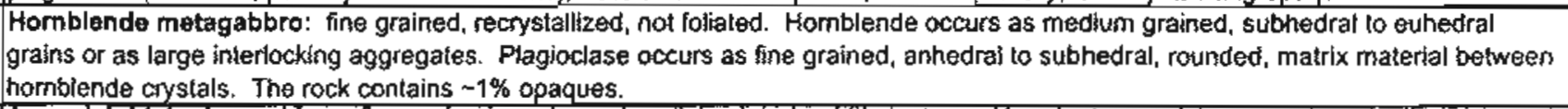 \\
\hline 99J\$23 & 387069 & & 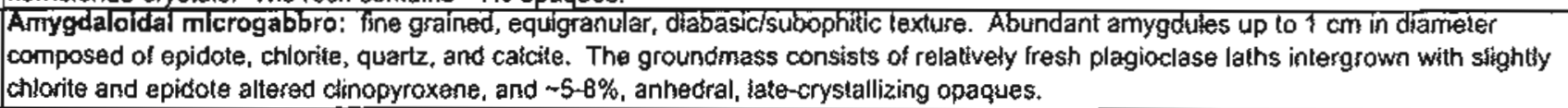 \\
\hline
\end{tabular}


Table 3. Location and description of rock samples collected in the Iron Creek area for major-oxide, minor oxide, and trace element analyses.

\begin{tabular}{|c|c|c|c|}
\hline Sample \# & UTME & UTM N & Sample description \\
\hline $99 J 525 \mathrm{~A}$ & 382110 & 6909272 & $\begin{array}{l}\text { Trondhjemlte: fight green, medium to coarse grained, equigramular. Primary minerals include quartz and plagiociase. Quartz occurs } \\
\text { as large anhedral grains, and quite commonly as mymekitic intergrowths with plagioclase. The rest of the plagioclase is subhedral to } \\
\text { anhedral and }+l \text {-albite twinned. No primary mafic minerals are present; they have been allered to patches of chlorite and secondary } \\
\text { opaques. Most of the rock is fresh but rare tiny fractures conlain sericite. }\end{array}$ \\
\hline 99JS26 & 386119 & 6909280 & 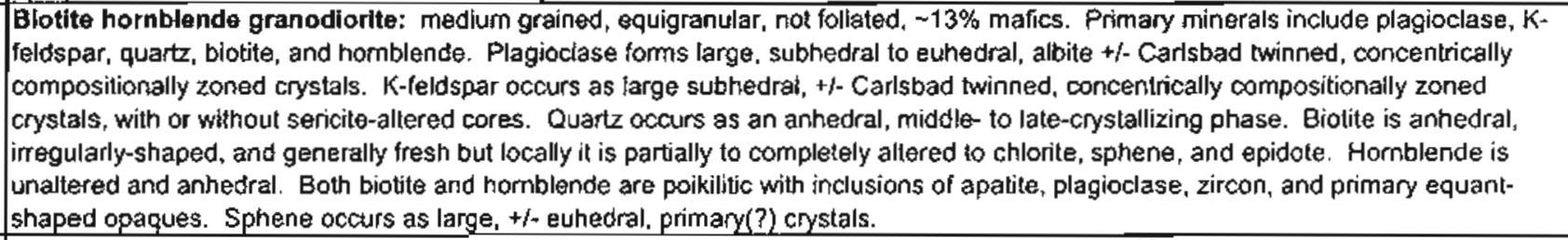 \\
\hline 99.JS28A & 393523 & 6912162 & $\begin{array}{l}\text { Hormblende tonalite: green and white, medium grained, equigranular, foliated/metamorphosed, }-25-30 \% \text { mafics. Primary minerals } \\
\text { include plagioclase, quartz, homblende, and minor bioute and opaques. Plagioclase occurs as large, anhedral to euhedral, albite } \\
\text { twinned, +/- deformed crystals that are extensively altered to epidote, sericite, and clay(?). Quartz occurs as large, anhedral, strained. } \\
\text { polycrystaline grains. Homblende occurs as large, subhedral crystals that are partially attered to chlorite, calcile, epiofole. and } \\
\text { secondary sphene. Biolite is altered to chlorite. epidote and sphene. There are small bands of more inlense strainfoliation that destroy } \\
\text { the original texture. }\end{array}$ \\
\hline 99JS2BF & 393523 & 6912162 & $\begin{array}{l}\text { Mudstone with quartz pebbles: pale gray. foliated. The mudstone is composed of mixed, tiny, angular to rounded grains of quartz and } \\
\text { chert floating in a very fine grained matrix of siliceous argillite(?) with }-1 \% \text { tiny, disseminated pyritefimonite grains. One microfraclure } \\
\text { cutting foliation contains sericite and calcile. }\end{array}$ \\
\hline $99 J 533 \mathrm{~A}$ & 393643 & 6921913 & $\begin{array}{l}\text { Cllnopyroxene gabbro: medium grained, equigranular, very altered. Primary minerals include plagioclase and clinopyroxene. } \\
\text { Plagioclase occurs as large, euhedral to subhedral, albite twinned, unzoned crystals. Plagioclase is intergrown with clinopyroxene, } \\
\text { which is partially altered to lelted masses of actinolite, minor chlorite, and trace tiny grains of limonite (afler secondary opacues(?)). No } \\
\text { primary opagues are present. }\end{array}$ \\
\hline $99 J \$ 36 \mathrm{~A}$ & 393773 & 6918282 & $\begin{array}{l}\text { Amygda loidal microgabbro: dark grayish green with diabasic(?) texture, rare glomeroporphyritic plagioclase phenocrysts, late- } \\
\text { crystalizing opaques, clinopyroxene (partially altered to chloritelpumpellyite(?)). Amygdules are filed with epidote, chlorite, } \\
\text { pumpellyite?, and (or) quarz. Rock is cut by microveinlet of unaltered plagioclase (albite(?)). }\end{array}$ \\
\hline 99J\$40A & 395639 & 6920792 & $\begin{array}{l}\text { Mlcrogabbro: fine grained, equigranular, diabasic to subophitic(?) texture. Primary minerals include plagioclase, clinopyroxene, } \\
\text { hornblende(?), and rare opaques. Plagioclase occurs as euhedral to subhedral crystals that are partially altered to clay and epidote(?). } \\
\text { Clinopyroxene occurs as relatively unaltered, roundish, equant grains that are parlially altered to actinolite and secondary opaques. The } \\
\text { microgabbro is cut by thin chlorite veins }\end{array}$ \\
\hline 99.JS42 & 388384 & 6911246 & 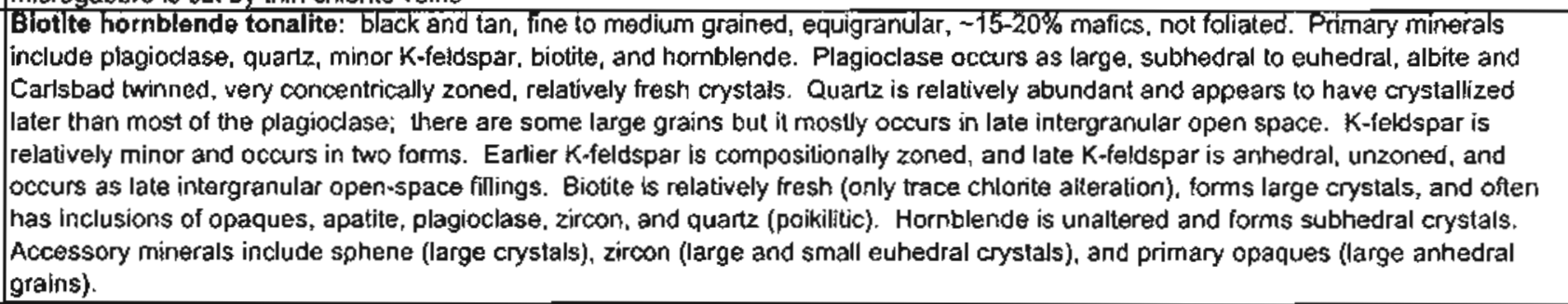 \\
\hline
\end{tabular}


Table 3. Location and description of rock samples collected in the Iron Creck area for major-oxide, minor oxide, and trace element analyses.

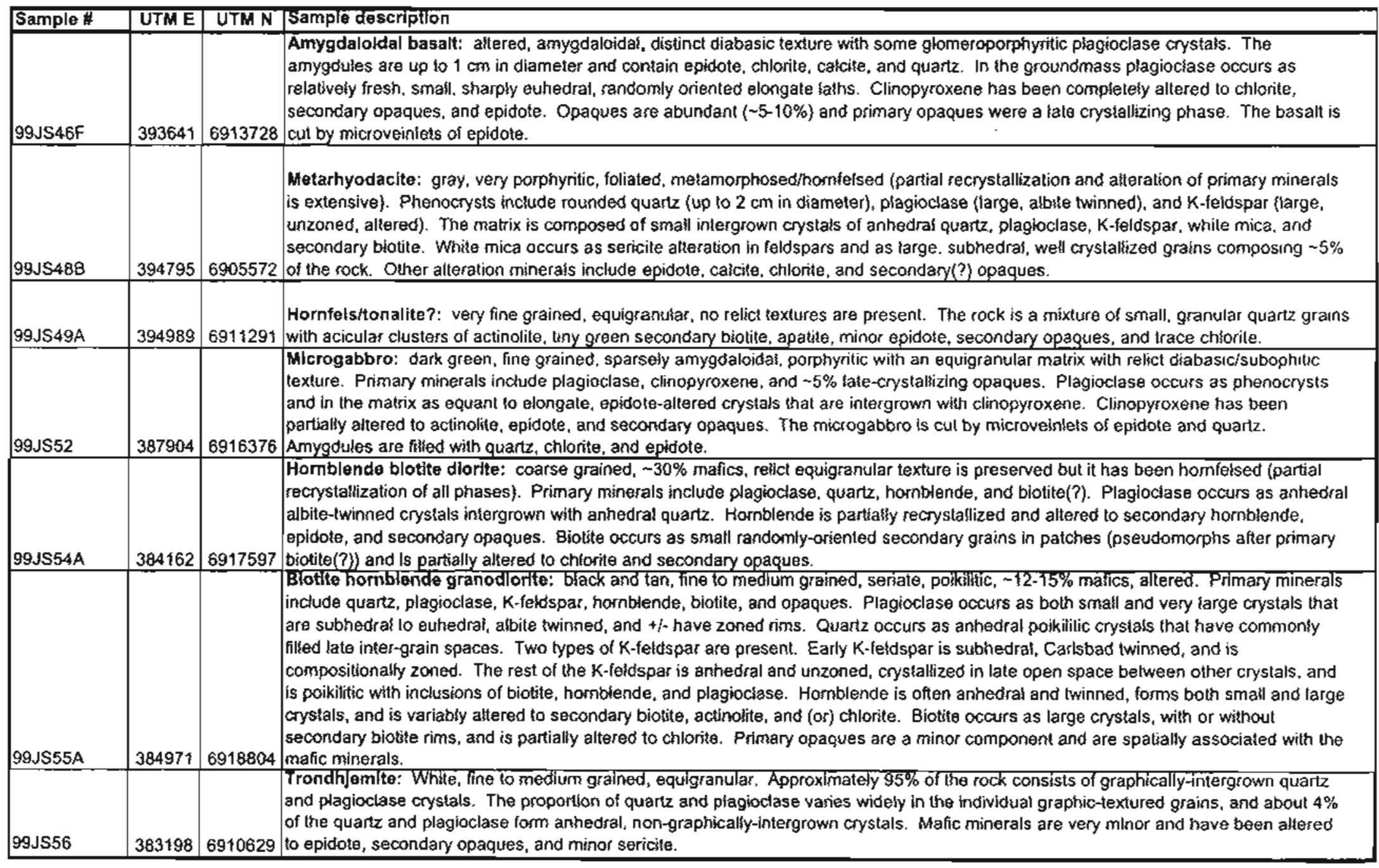


Table 3 . Location and description of rock samples collected in the Iron Creek area for majortoxide, minor oxide, and trace clement analyses.

\begin{tabular}{|c|c|c|c|}
\hline Sample \# & UTME & UTMN & Sample description \\
\hline $90 J 559 \mathrm{~A}$ & 389181 & 6909576 & 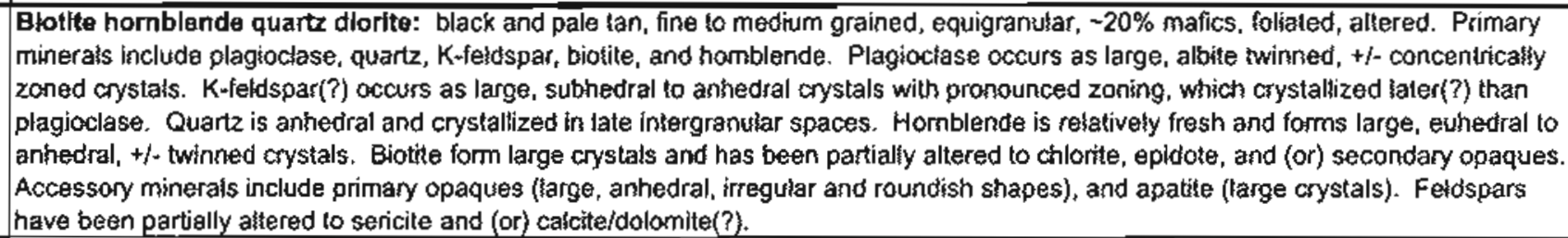 \\
\hline $99 \mathrm{~J} \$ 6) \mathrm{A}$ & 396148 & 6916488 & $\begin{array}{l}\text { Amygdaloldal basalt: extensively epidote aftered, poorty preserved relict equigranular texture, and relict glomeroporphyritic texture with } \\
\text { plagiociase phenocrysts. Amygdeles filted with chiorite, epidote, quariz, and minor calcite. Contains }-5 \% \text { disseminale, equant, }+1- \\
\text { irregularty-shaped primary(?) cpaques. }\end{array}$ \\
\hline 99.JS64A & 391639 & 6914967 & $\begin{array}{l}\text { Amygdaloldal basalt/microgabbro: fine grained, relict subophitic and glomeroporphyritic textufe. Primary minerals consist of } \\
\text { intergrown plagioclase, clinopyroxene. and } 3-5 \% \text { late-crystallizing opaques. Plagioclase occurs as phenocrysts and as randomly. } \\
\text { oriente, laths in the matrix with minor epidote alteration. Clinopyroxene is partially to completely altered to actinofite, chlorite. and } \\
\text { epidote. Amygdules are filted wilh chlorite, epidote. and minor quartz. }\end{array}$ \\
\hline 99MBW403 & 381545 & 6920029 & 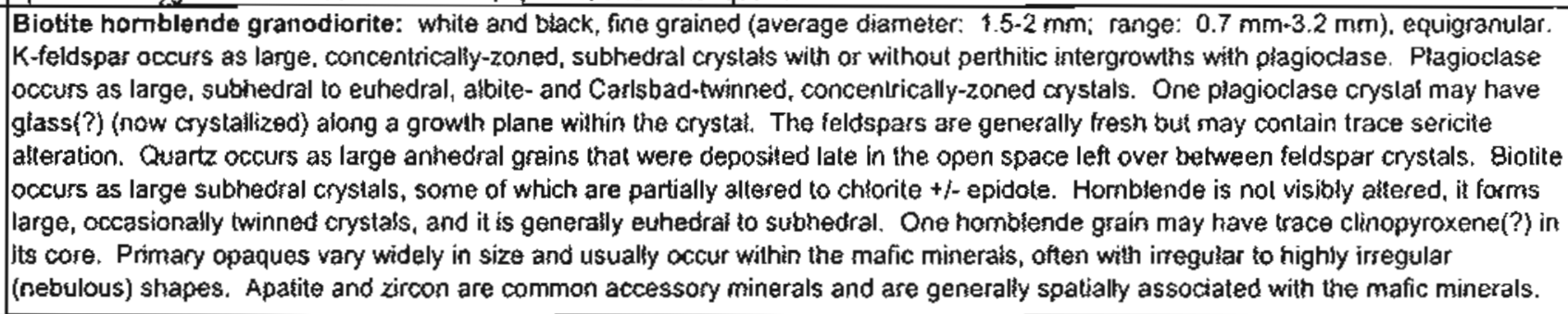 \\
\hline $99 \mathrm{MB} W 408 \mathrm{~A}$ & 380546 & 6920664 & $\begin{array}{l}\text { Hormfelsed volcanlclastlc braccia: medium gray, breccia fragments include: 1) medium grained plutonic rock fragmenis, and 2) } \\
\text { interlocking crystalline plagioclase fragments in a matrix of clastic(?) accumulations of quartz and plagioclase crystass. Metamorphic } \\
\text { minerak include epidole, acicular aclinolite(?) and disseminated iron sulfides. }\end{array}$ \\
\hline $99 \mathrm{MBW} 410 \mathrm{~B}$ & 380242 & 6921146 & Alkali granlte aplite dlke: white, Gne grained, anthedral granular texture; dike is $-10 \mathrm{~cm}$ wide. \\
\hline 99MBWA11 & 384675 & 6913498 & $\begin{array}{l}\text { Basaktlc andes ite: well-jointed massive outcrop; dark green, epidote aftered, aphanitic to very fine grained, equigsanular. Primary } \\
\text { minerals are not present. Melamorphic minerals include actinolite, clay (after plagioclase), secondary opaques, epidote, and calcite. }\end{array}$ \\
\hline 99MBW415 & 382324 & 6912556 & $\begin{array}{l}\text { Microgabbro: medium green, fine grained, equigranular, relict subophitic(?) texture, highly altered. The rock is composed of } \\
\text { plagioclase, epidote, trace iddingsite(?), quartz, calcite, and opaques. Epidote appears to have replaced clinopyroxene, and plagioclase } \\
\text { is partialiy replaced by epidole and chlorite. }\end{array}$ \\
\hline $99 M B W 416$ & 385275 & & $\begin{array}{l}\text { Porphyrtic metamorphosed microgabbro: medium to dark green, equigranular, very fine grained, blocky weathering, very allered. } \\
\text { Relict subophitic(?) and glomeroporphyritic textures are variably preserved. Metamorphic minerals include abundant epidote, actinolite, } \\
\text { chlorite, catcite, and opaques. Plagkoclase occurs in glomeroporphyric clusters. }\end{array}$ \\
\hline
\end{tabular}




\begin{tabular}{|c|c|c|c|}
\hline Sample \# & UTME & UTM N & Sample description \\
\hline $99 \mathrm{MBW} 418 \mathrm{~B}$ & 387061 & 6910235 & $\begin{array}{l}\text { Hornblende-biotite tonalite: gray, fine grained, equigranular, slightly altered. Plagioclase occurs as large. Cartsbad-and albite- } \\
\text { twinned, concentricalty-zoned crystals, some of which have thin rims of unzoned, untwinneo feldspar. K-feldspar is relatively minor and } \\
\text { foms untwirned, concentrically zoned crystals. Feldspars are vatiably altered by minor sericite and (or) calcite. Quartz occurs as large } \\
\text { anhedral crystals, and as myrmekitic intergrowths with feldspar. Homblende is relatively unaltered but biotite is partially to completely } \\
\text { altered to chlorite. Apatite and primary opaques form relatively large grains, and most commoniy are spatially associated with mafic } \\
\text { minerals. Large, homb-shaped crystals of sphene(?) (or perhaps dolomite(?)) are common throughout the lonalite. }\end{array}$ \\
\hline $99 M B W 419 C$ & 395784 & 6914903 & Blottte granodlorlte/tonalite: Tan weathering, light colored, fine grained, equigranular, quartz-rich $(>20 \%),-7 \%$ biotte. \\
\hline $99 \mathrm{MBW} 421$ & 395707 & 6914685 & $\begin{array}{l}\text { Gabbro: medium lo dark green, fine grained, highly magnetic, epidote altered along joints. Primary mineraks include plagioclase, } \\
\text { clinopyroxene, and late-crystallizing opaques. The plagioclase and clinopyroxene are intergrown with an equigranular subophitic texture. } \\
\text { Metamorphic minerals include chlorite, epldate, actinolite(7), pumpellyite(?), opaques, and trace calcile and quartz; tiny roundish } \\
\text { patches (arnygdules?) are predominantly filled with chlorte, and lessor epidote and pumpellyile(?). }\end{array}$ \\
\hline 99MBW/422 & 395497 & 6914783 & $\begin{array}{l}\text { Amygdaloidal metagabbro: dark to medium green, amygdules filied wilh epidote, chlorite, and milky, feathery-textured quartz The } \\
\text { matrix has a relict subophitic texture. Plagioclase crystals are fairly fresh, clinopyroxene has been replaced by actinolite, and primary } \\
\text { opaques crystallized late in intergranular spaces. Epidole alteration of the groundmass is extensive. The melagabbro is cut by } \\
\text { anastomosing foliatoon bands thal contain broken metagabbro fragments. }\end{array}$ \\
\hline 99MBW435 & 394739 & 6914976 & $\begin{array}{l}\text { Metagabbro: green, equigranular to slightly porphyritic(?). Primary minerals include plagloclase, opaques, and probably clinopyroxene. } \\
\text { Primary opaques crystallized in late open intergranular space. Metamorphism has resulted in extensive replacement of primary minerals } \\
\text { by actinolite, calcite, epidote, chlorite, and secondary opagues. }\end{array}$ \\
\hline 99MBW440 & 392358 & 6919922 & $\begin{array}{l}\text { Olivine microgabbrolpicrite basalt: Dark brownish-black, very fine grained, rare amygdules filled with calcite and (or) quartz. The } \\
\text { matrix predominantly has an equigranular, diabasic, subophtitc lexture, with sparse local ophitic texture. Primary minerals include } \\
\text { unaltered plagioclase laths, olivine ( } 35 \% \text {; completely pseudomorphed by iddingsite }+/ \text { - calcite), clinopyroxene, questionable } \\
\text { orthopyroxene(?), and late-crystallizing opaques. Caldie occurs in thin cross-cutting veins. }\end{array}$ \\
\hline $99 \mathrm{MBW} 441 \mathrm{~A}$ & 392469 & 6920076 & $\begin{array}{l}\text { Dacíte dlke: gray and white, seriate-textured, large phenocrysts of plagloclase, K-feldspar, quartz, and blotite, The matrix is almost } \\
\text { completely crystalline and consists of feldspars, quartz, chloritized biotite, apalite, and tiny opaque minerats. Alteration includes } \\
\text { recrystallization of quartz phenocrysts, clay (afler feldspars), sericite (after feldspars and biotite), and epidote and chlorite (after biotite). }\end{array}$ \\
\hline 99MBW450 & $3928+6$ & 6919823 & $\begin{array}{l}\text { Blotite tonalite: while, medium grained, porphyntic. Phenocrysts tnclude large plagioclase and quartz phenocrysis (up to } 1 \text { cm on } \\
\text { average) in a medlum gray matrix. The quartz phenocrysts have been strained and partially recrystallized. Plagioclase occurs as } \\
\text { subhedral, abite twinned, +//-concentrically zoned crystals. K-felidspar occurs as perthite and as concentrically zoned crystals linat are } \\
\text { clay altered in their cores. Biotite occurs throughout the groundmass as large primary crystals surrounded by margins of smaller } \\
\text { secondary biotite. The biotite has also been partially altered to chlorite, sericite, epidote, secondary opaques and (or) calcite. } \\
\text { Accessory minerals include apatite and large primary opaques. }\end{array}$ \\
\hline $99 \mathrm{MBW} 453$ & 393240 & 6922624 & $\begin{array}{l}\text { Granite pegmatite: quartz-rich, miedium grained, roughly equigranular. Primary minerals include quartz. K-feidspar, plagloclase, and } \\
\text { fluorite. Quartz occurs as large and small anhedral crystals, with some of the larger grains exhibiting undulatory extinction and partial } \\
\text { recrystailization. K-feldspar is present as large, anhedral perthite crystals. Plagioclase occurs as smaller, albite winned, subhedral to } \\
\text { anhedral crystals. Fluorite occurs as anhedral grains deposited in late inter-crystalline spaces. The pegmatle is altered, and sericite } \\
\text { occurs as tiny crystals replacting feldspars and along microfractures and grain boundaries. Trace epidote and secondary(?) opaques are } \\
\text { also present. }\end{array}$ \\
\hline $99 \mathrm{MB} W 456$ & 393090 & 6922884 & $\begin{array}{l}\text { Altered granite: orange-weathering, iron-stained with leisegang banding. massive, jointed outcrop. Pluton is pink, medium grained, } \\
\text { equigranutar, and very altered. Primary minerals include quartz, plagioclase, K-feldspar, biobte(?). Quartz is abundant (up to } 40 \% \text { ), } \\
\text { structurally deformed, and occurs as recrystallized anhedral grains. K-foldspar occurs as perthitic intergrowths with plagioclase. } \\
\text { Plagioclase also occurs as separate, abite twinned crystals. Mafic minerals (probably former biotite) have been replaced by sericite. } \\
\text { epidote, and secondary opaques. Most feldspars are relatively unaltered but some contain trace sericite alteration. }\end{array}$ \\
\hline
\end{tabular}


Table 3. Location and description of rock samples collected in the Iron Creek area for major-oxide, minor oxide, and trace element analyses. \begin{tabular}{l|l|l|l}
\hline Sample \# & UTM E & UTM N & Sample descrpton
\end{tabular}

\begin{tabular}{|c|c|c|c|}
\hline 99MBW459 & 393913 & 6922603 & $\begin{array}{l}\text { Diorite: medium green, fine to medium grained, equigranular, }-40 \% \text { dark green clinopyroxene(?) and }-60 \% \text { plagioclase with minor } \\
\text { epidote and chlorite(?) alteration. }\end{array}$ \\
\hline 99NEW & 394418 & 6922294 & $\begin{array}{l}\text { Dlorite: dark green and white, medium grained, approximately equigranular, contains }-40 \% \text { randomly oriented, elongate, euhedral laths } \\
\text { of homblende floating in a matrix of white plagioclase }(-60 \%) \text { that are partially altered to epidote. }\end{array}$ \\
\hline $99 \mathrm{MBW} 477$ & 394632 & 6921815 & $\begin{array}{l}\text { Metabasalt/graenstone: medium green, aphanitic, unioliated rock with no relict primary textures. Composed of fresh-looking, small } \\
\text { elongate laths of plagioclase randomly orlented in a slightly finer grained matrix of randomly oriented pumpellyite(?) and opaques. Rock } \\
\text { is cut by veins of albite, clinozoisite, pumpelyyile(?), quartz, chlorite, and (or) limonile. }\end{array}$ \\
\hline $99 \mathrm{MB} W 480$ & 394688 & 6921746 & $\begin{array}{l}\text { Porphyritc amygdaloidal basalt: green, fine grained, highly altered, relic diabasic/subophitic texture. Primary minerals include } \\
\text { elongate-shaped, +1-twinned clinopyroxene intergrown with untwinned. clay-altered plagioclase, and tiny disseminated opaques. } \\
\text { Amygdules are filled with chlorile, actmolite, calcite, and (or) epidote. Metamorphic minerals in the groundmass include epidote, clay, } \\
\text { chlorite, and calcite, and the groundmass exhibits patchy areas of relatively unaltered and extensively allered material. }\end{array}$ \\
\hline S9MBW4B1 & 394726 & 6921742 & Granite? dike or plug?: while to pale pink weathering, +/- jron-stained, quarlz-rich, equigranular, fine to medium grained. \\
\hline 99MBW485 & 394915 & 6921673 & $\begin{array}{l}\text { Metamorphosed microgabbro: dark green, very fine grained, porphyntic. Plagioclase phenocrysts are glomeroporphyritic, and occur } \\
\text { throughout a subophitic matrix of intergrown plagloclase, clinopyroxene, and late-crystallizing opaques. Metamorphic alteration minerals } \\
\text { include epidote, calcite, and abite( } 7) \text {. There is extensive epidote alteration of the matrix and plagioclase phenocrysts. Albite(?) occurs } \\
\text { as small, relatively fresh crystals in the matrix. Calcite occurs in thin velns. }\end{array}$ \\
\hline 99MBW487 & 394966 & 6921661 & $\begin{array}{l}\text { Metagabbro: homfelsed, pale green, fine gratned, equigranular. Primary minerals include plagioclase, clinopyroxene, and euhedral } \\
\text { apatite. The rock is largely composed of plagioclase, which occurs as large relict interlocking bladed crystals that have been extensively } \\
\text { replaced by epidote and calcite. Relict clinopyroxene cystals are commonly twinned and are much smaller than the plagiockase } \\
\text { crystals. Metamonhic minerals include clinozoisite, epidote, calcite, tremolite, and trace quarlz. Opaque minerals are not present. }\end{array}$ \\
\hline 99MBW492 & 395621 & 6921528 & $\begin{array}{l}\text { Trondhjemite: pale gray, equigranular, fine grained. Primary minerals include quartz (>20\%), and plagioclase. Quartz occurs as } \\
\text { anhedral grains, most of which cryslallized later than plagioclase. Plagioclase occurs as euhedral ta subhedral, albite and Carlsbad } \\
\text { lwinned crystals. Alteration minerals include epidote (abundant, replaces piagioclase), chlorte (after biotite?), and caicite (in } \\
\text { microfractures). Large opaques (possibly primary?) have been partially removed or redistributed within the rock during afteration, }\end{array}$ \\
\hline 99MBW493 & 395780 & 6920916 & $\begin{array}{l}\text { Basalt/basaltic andesite: brown weathering. massive, minor epidote and red hematite on fractures in oulcrop. Most of the rock is } \\
\text { composed of a randomly oriented mixture of euhedral to subhedral plagioclase laths and actinolite (probably replaced clinopyroxene). } \\
\text { The textures appears equigranular and may have been subophitic(?). Metamorphic minerals include actinolite, epidole (replacing } \\
\text { plagioclase and pyroxene), trace quartz, and leached, limonite altered opaques. }\end{array}$ \\
\hline $99 M B W 494$ & 390358 & 6918894 & $\begin{array}{l}\text { Amygdaloidal basalt: medium green, amygdules filled with quartz and acicular sprays of epldote. Outcrop cut by epidole veins. In thin } \\
\text { section the basalt is extremely altered and primary matrix textures are not preserved. Relict primary plagioclase phenocrysts occur in } \\
\text { glomeroporphyritic clusters, and are partially altered to calcite and epidote. Metamorphic minerals include epidole, chlorite, calcite. } \\
\text { actinolite, and pumpellyite(?). }\end{array}$ \\
\hline 99MBW50O & 390615 & 6918385 & $\begin{array}{l}\text { Metabasalt: medium to dark green, very fine gralned, massive, amygdaloidal. Amygdules contain chlorite and epidote and are up to } 2 \\
\text { cm in diameler. }\end{array}$ \\
\hline $99 \mathrm{MBW}$ 506 & 379284 & 113910 & $\begin{array}{l}\text { Hornblende diorltefgabbro: medium gray, fine to medium grained, appears equigranular bul contains large poikilitic hornblende } \\
\text { crystals }(-25-30 \%) \text { up to } 2.5 \mathrm{~cm} \text { in diameter. Plagloclase may be slightly epidote altered and composes }-70-75 \% \text { of the rock. }\end{array}$ \\
\hline
\end{tabular}


Table 3. Location and description of rock samples collected in the Jron Creck area for major-oxide, minor oxide, and trace element analyses.

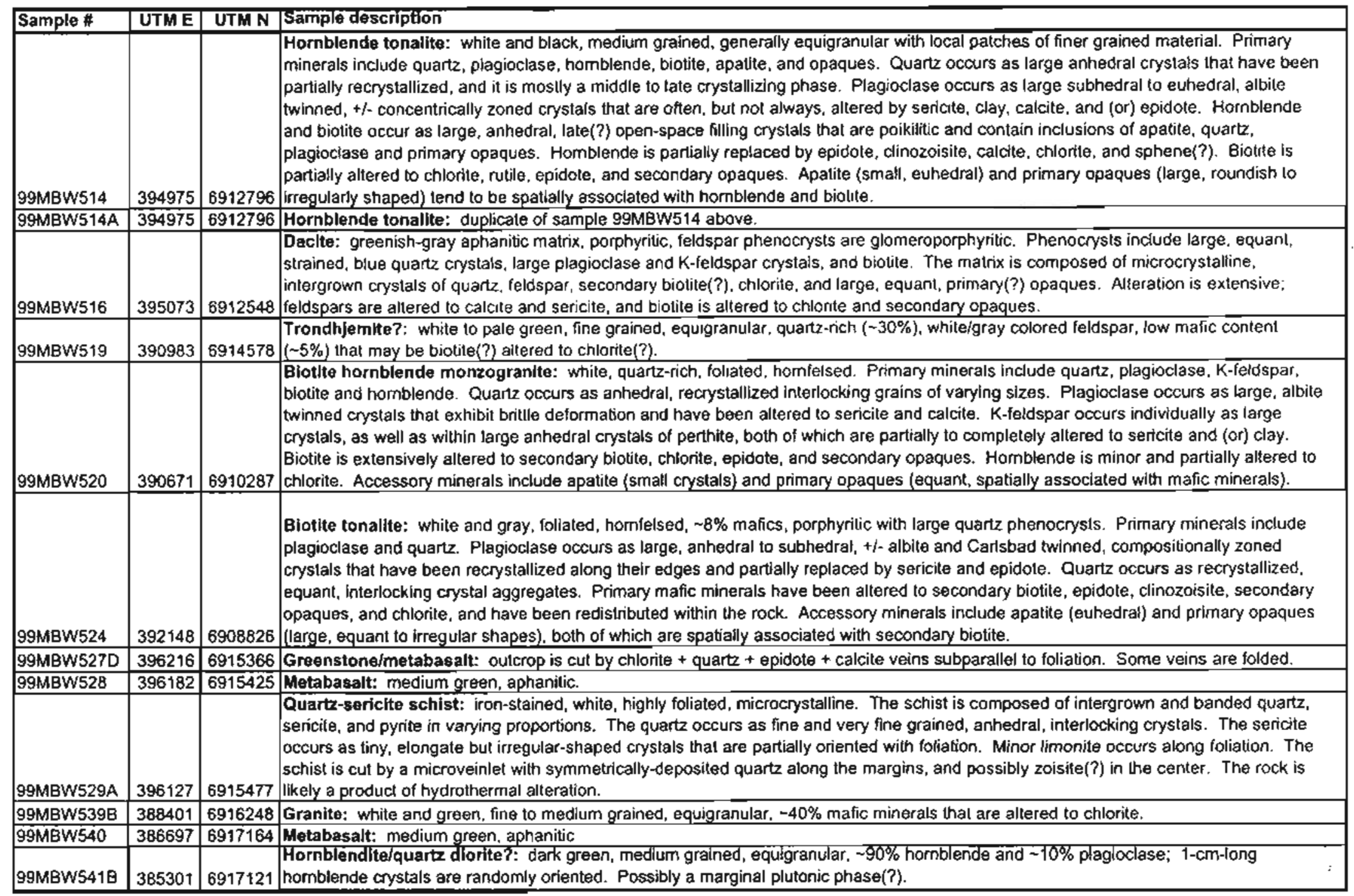


Table 3. Location and description of rock samples collected in the Iron Creek area for major-oxide, minor oxide, and trace clement analyses.

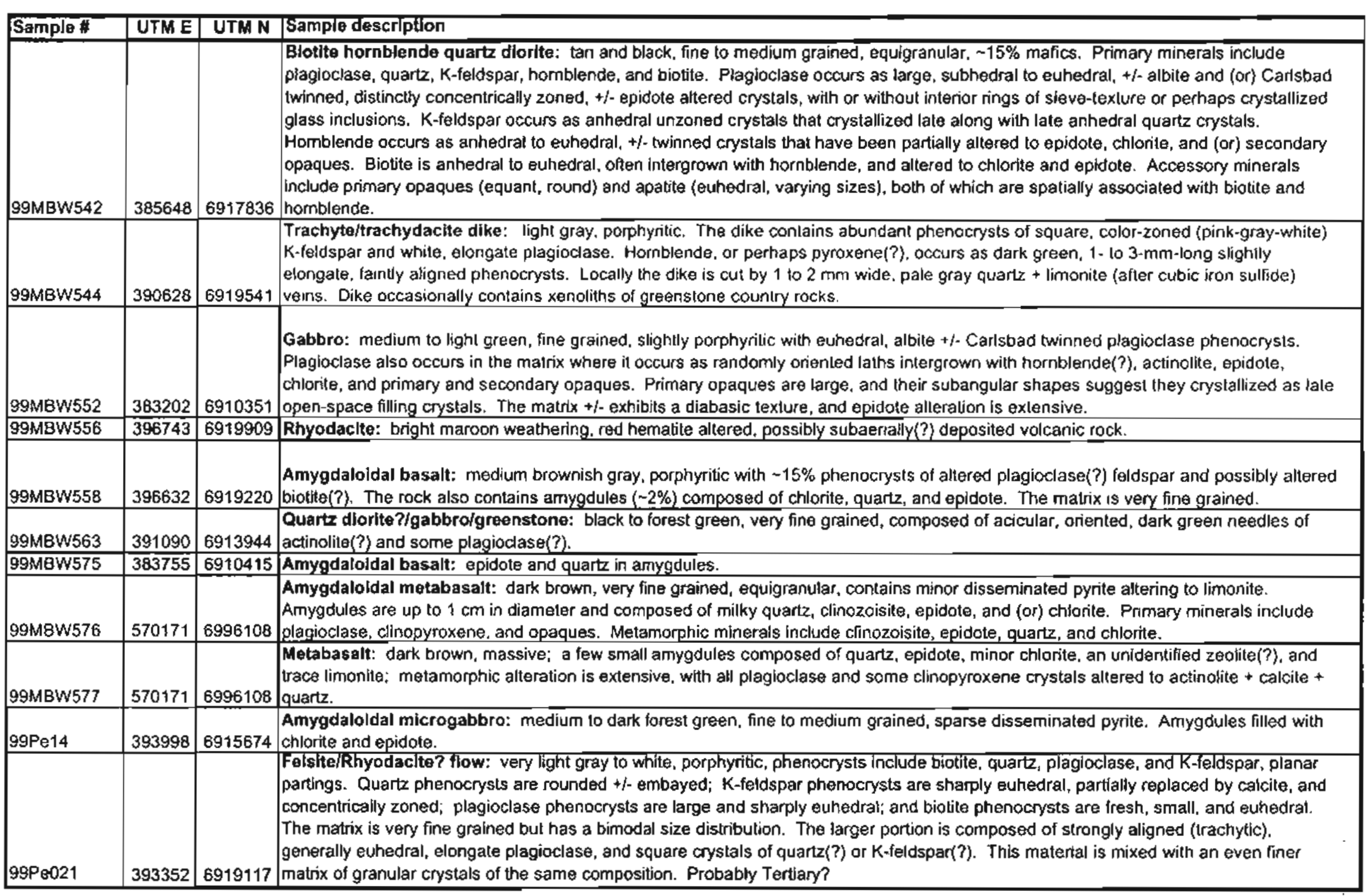




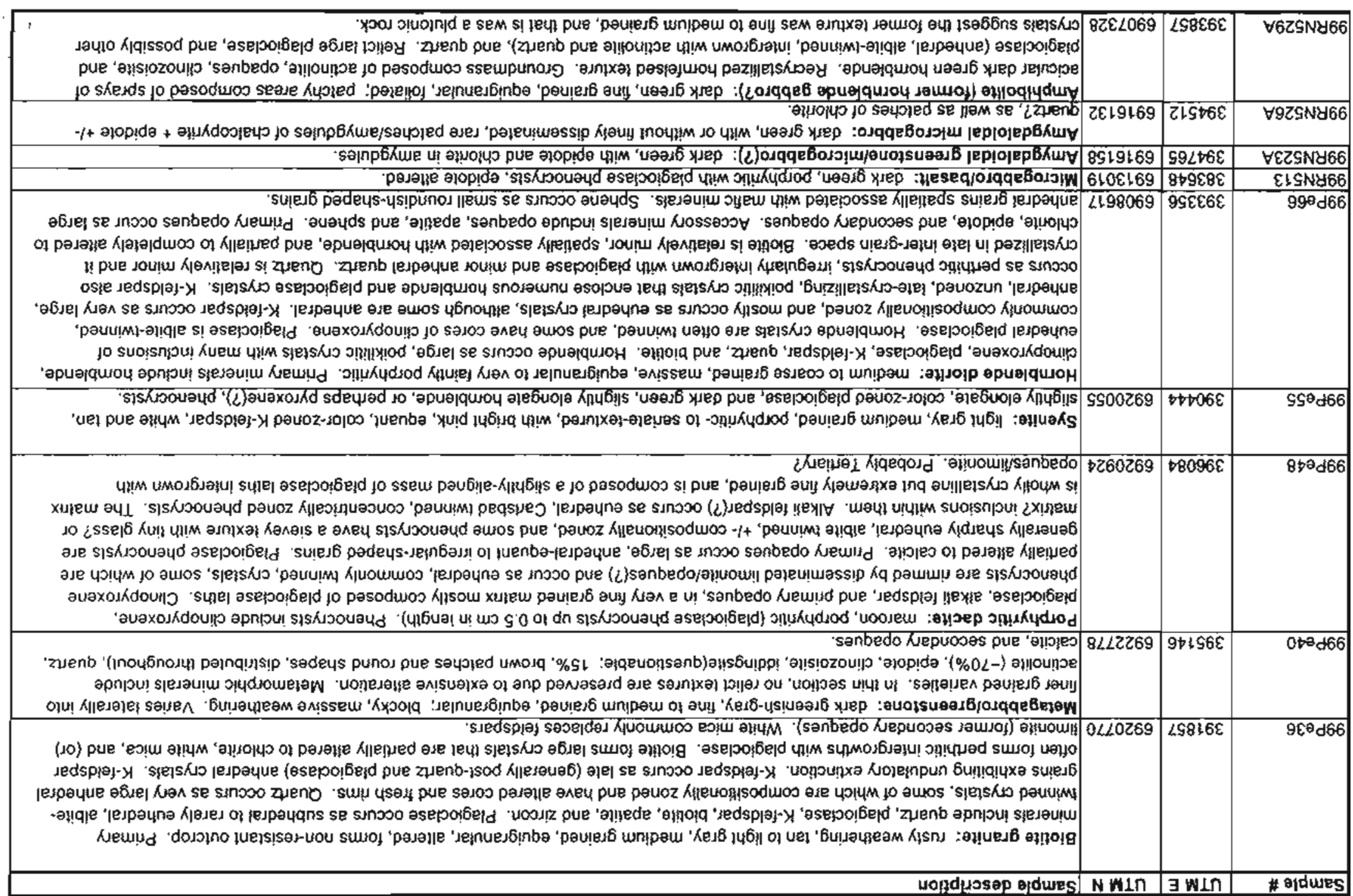

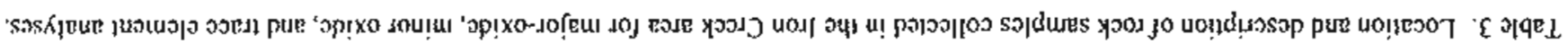


Table 3. Location and description of rock samples collected in the lron Creek area for major-oxide, minor oxide, and trace element analyses.

\begin{tabular}{|c|c|c|c|}
\hline Sample \# & UTM E & UTH N & Sample descrpton \\
\hline 99RN530A & 393339 & 6906715 & 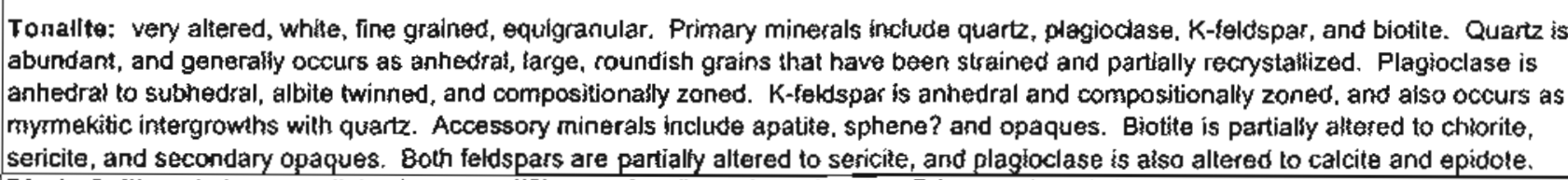 \\
\hline 99RN530B & 393339 & & 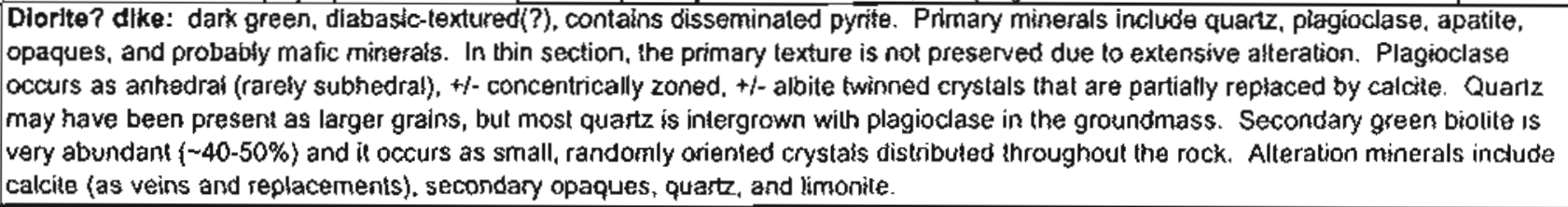 \\
\hline
\end{tabular}


Table 4. Concentration of major-oxides, minor oxides, and Irace elements in rock samples from the Iron Creek area.

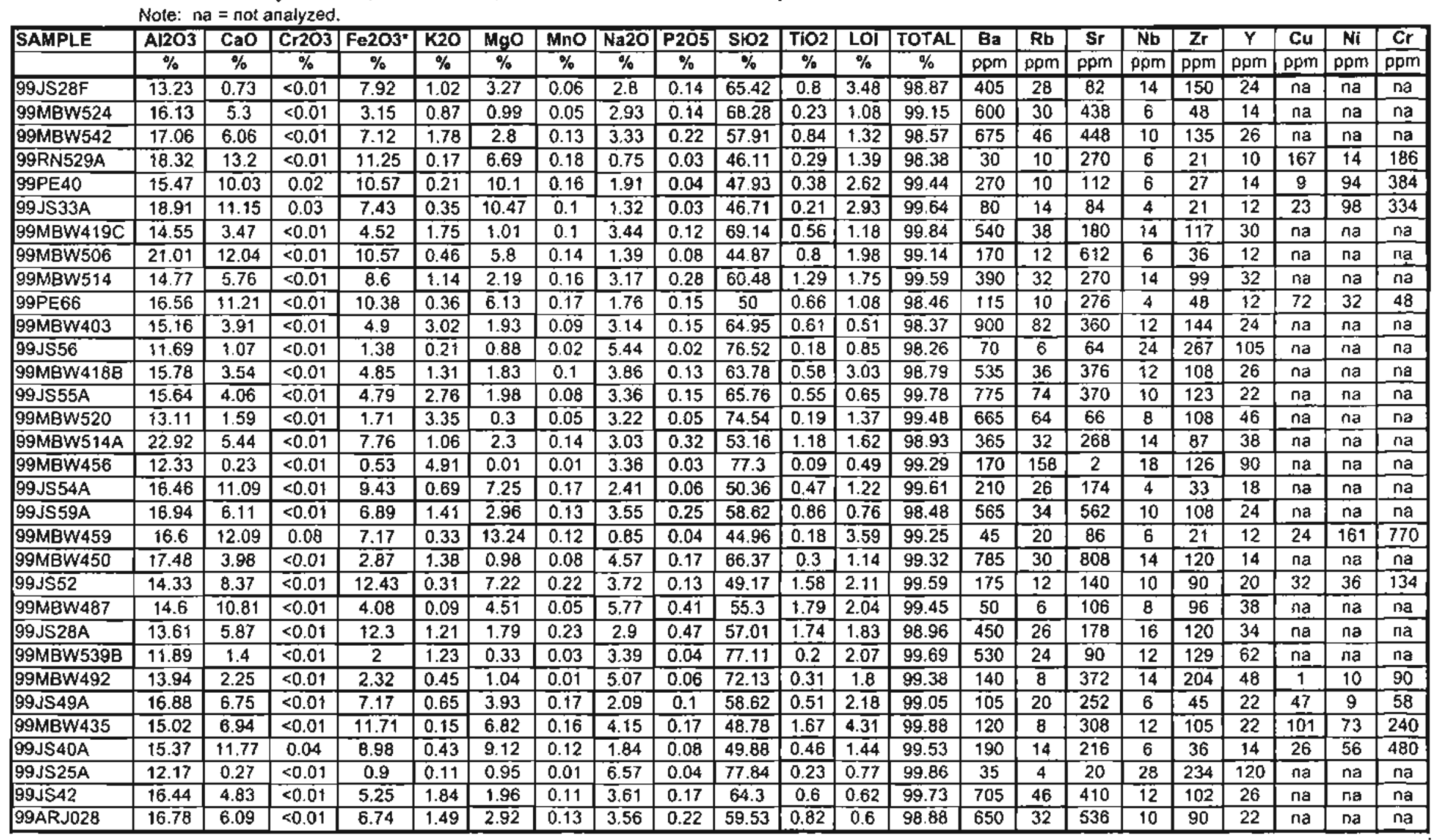


Table 4. Concentration of major-oxides and trace elements in rocks from the Iron Creek area, Talkectna Mountains (B-5) quadrangle.

Note: na $=$ not analyzed.

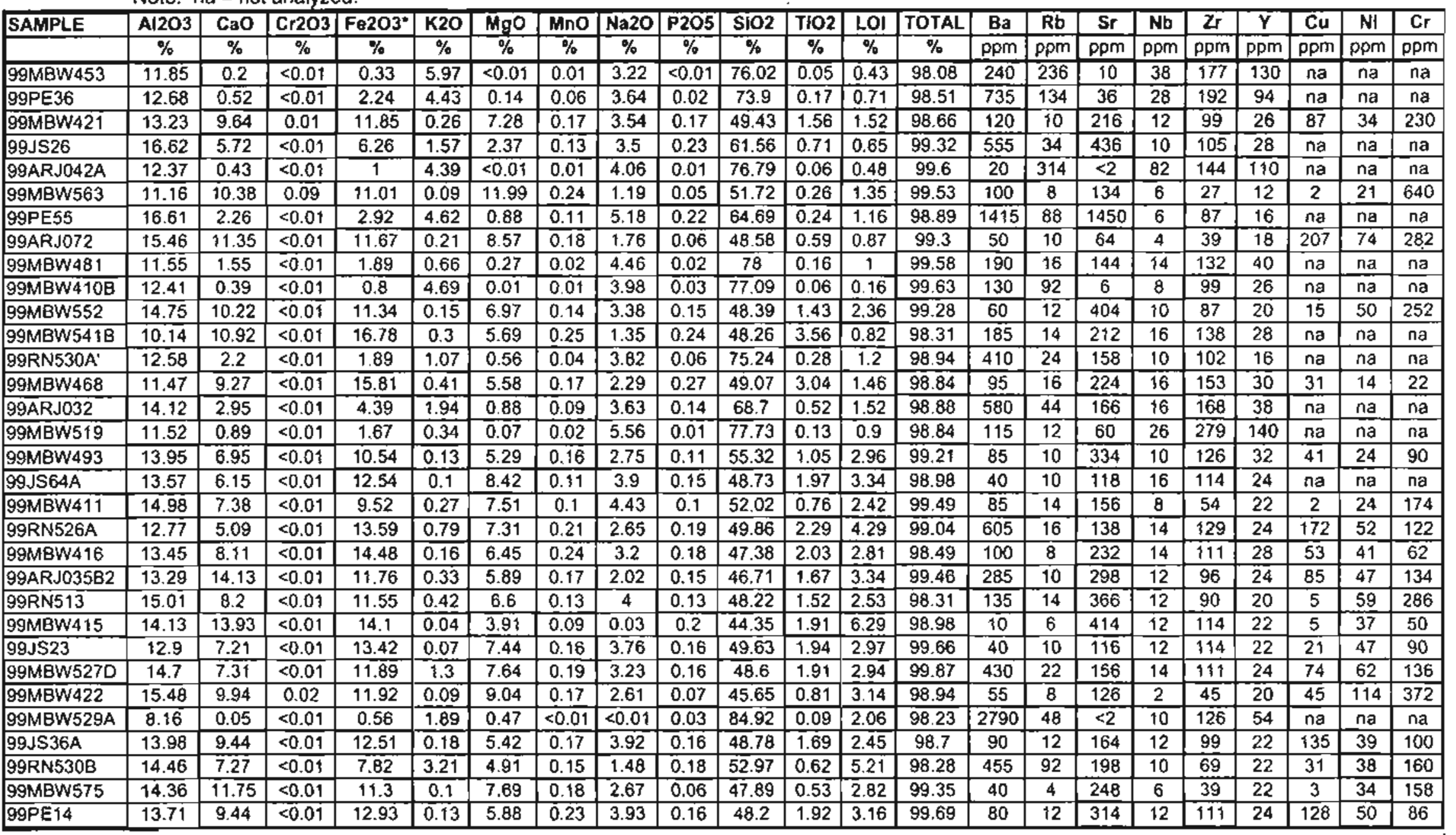




\begin{tabular}{|c|c|c|c|c|c|c|c|c|c|c|c|c|c|c|c|c|c|c|c|c|}
\hline$\overline{0}$ & 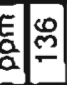 & $\stackrel{0}{\sim}$ & 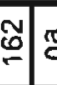 & $\begin{array}{ll}0 \\
= \\
0\end{array}$ & & & $\mathscr{E}$ & ת) & & 0 & & & $\stackrel{\mathbb{g}}{c}$ & $\delta$ & & & & & & $\mathscr{0}$ \\
\hline & $\bar{a} \mathbf{a} \mid \bar{v}$ & 10 & 8 & 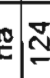 & & & & & & & $\infty$ & ज्ञ & $\mathbb{E}$ & & 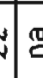 & & 아 & & & ஜ \\
\hline 己े & & & go & $g$ & $\mid \begin{array}{l}\tilde{N} \\
\underline{m}\end{array}$ & $\mathbb{g}$ & & 98 & & 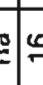 & $\approx$ & $\Xi$ & & & & & $\mid$ & $\mathbb{5}$ & & $\stackrel{\pi}{c}$ \\
\hline$>\mid$ & $\begin{array}{c}\bar{c} \\
\bar{c}\end{array}$ & & & 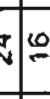 & N & & & & & 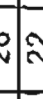 & & $\mathbf{N}$ & $\begin{array}{l}0 \\
\text { ஸी }\end{array}$ & & & $\pi$ & $\mathbb{N}$ & \pm & & $\stackrel{\infty}{\sim}$ \\
\hline & $\begin{array}{c}\mathbf{2} \\
\alpha\end{array}$ & & $\infty$ & $\overline{0}$ & $=$ & & $\stackrel{g}{\circ}$ & $\frac{x}{2}$ & & 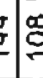 & D & $\frac{\sigma}{\sigma}$ & 商 & 3 & & 8 & $\approx$ & $\vec{B}$ & & 这 \\
\hline 妾 & $\begin{array}{c}\mathbf{E} \\
\mathbf{2}\end{array}$ & $\sim \subseteq$ & $\circ \infty$ & $0 \infty$ & $\mathbb{E}$ & $\infty$ & $\Phi$ & v. & $0 \leqslant$ & $2:=$ & $\sigma$ & $\approx$ & $\stackrel{\infty}{\sim}$ & 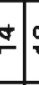 & & $\infty$ & $\approx$ & $\mathbb{F}$ & & o \\
\hline$E$ & $\bar{a} \mid \mathbb{a}$ & $\stackrel{0}{\sim} \underset{0}{\infty}$ & $\stackrel{ \pm}{ \pm}$ & 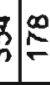 & $\stackrel{8}{\circ}$ & & 声 & & & & $\vec{N}$ & 竞 & 웜 & 8 & & $\mathbb{\infty}$ & 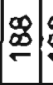 & & 品 & $\stackrel{\varrho}{\rightleftharpoons}$ \\
\hline & & & & $\underset{N}{J}$ & 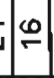 & 이 & $\approx$ & $\theta$. & 0 s & $\infty$ & 오 & I্র & $\mathbb{\infty}$ & D & & O্ল & 응 & 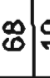 & & $\mathbb{N}$ \\
\hline$\approx$ & E⿱ & ஜூ & 兽) & $\frac{\Omega}{\sigma}$ & 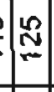 & & 邑 & 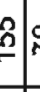 & & 8 & & 跑 & 品 & & & 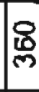 & 인 & 号 & & $\stackrel{s}{=}$ \\
\hline $\begin{array}{l}\frac{1}{0} \\
\\
0\end{array}$ & $=\left[\begin{array}{l}\bar{n} \\
0 \\
0\end{array}\right.$ & $\begin{array}{ll}0 \\
0 \\
g \\
g\end{array}$ & 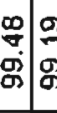 & 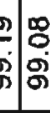 & 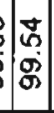 & \begin{tabular}{l}
0 \\
\hdashline \\
8 \\
\hdashline
\end{tabular} & & & & 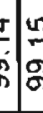 & $\begin{array}{l}n \\
\alpha \\
\alpha \\
\sigma\end{array}$ & $\begin{array}{l}m \\
3 \\
\infty \\
\infty\end{array}$ & 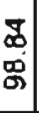 & 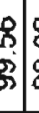 & & 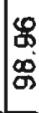 & $\left.\mid \begin{array}{l}0 \\
0 \\
0 \\
0 \\
0\end{array}\right]$ & 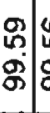 & & \\
\hline & 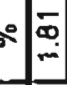 & ra & \begin{tabular}{c|c}
3 & 5 \\
6 & 5
\end{tabular} & 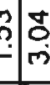 & $\stackrel{g}{\stackrel{g}{-}}$ & & & & & $\bar{r}$ & $\stackrel{n}{n}$ & \begin{tabular}{|c|}
$\tilde{\sigma}$ \\
$\dot{m}$ \\
\end{tabular} & 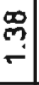 & 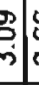 & & $\stackrel{m}{-}$ & 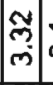 & $\bar{m}$ & & 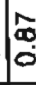 \\
\hline O气 & 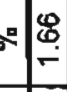 & $\cong$ & 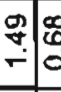 & $\begin{array}{l}8 \\
0 \\
:\end{array}$ & $\frac{n}{\bar{N}}$ & 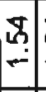 & 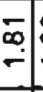 & 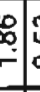 & & $\left.\begin{array}{c}0 \\
\vdots \\
\vdots\end{array}\right]$ & $\pi$ & 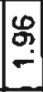 & $\begin{array}{l}\infty \\
\vdots \\
-\end{array}$ & $=$ & $=\sqrt{2}$ & ্ָ & $\begin{array}{lll}F & \\
r & 0\end{array}$ & & $=0$ & \\
\hline :̊ & 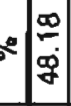 & \begin{tabular}{ll}
$\infty$ \\
\hdashline \\
$\dot{y}$
\end{tabular} & 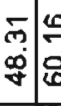 & \begin{tabular}{l|l}
0 & 1 \\
0 \\
0
\end{tabular} & {$\left[\begin{array}{l}0 \\
0 \\
0 \\
0\end{array}\right.$} & 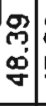 & 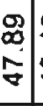 & $\begin{array}{ll}0 \\
8 \\
8 \\
8\end{array}$ & & $\bar{z}$ & & 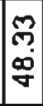 & $\begin{array}{l}\mathbb{X} \\
\dot{J}\end{array}$ & $\bar{\tau}$. & & $\frac{\text { N }}{4}$ & $\mid$ & & & \\
\hline $\begin{array}{ll}0 \\
0 \\
0\end{array}$ & $2 \frac{20}{0}$ & 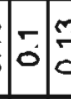 & \begin{tabular}{c|c}
$\frac{m}{0}$ \\
\end{tabular} & $=0$ & $\frac{9}{0}$ & $=$ & & $\begin{array}{l}0 \\
0 \\
0\end{array}$ & 2 & & 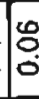 & $\frac{9}{0}$ & $\begin{array}{l}n \\
0 \\
0\end{array}$ & 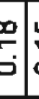 & & 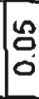 & $\mid \begin{array}{c}0 \\
0 \\
0\end{array}$ & $\overline{0}:$ & & \\
\hline 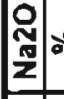 & & $\begin{array}{c}\stackrel{N}{\sim} \\
\text { id }\end{array}$ & $\bar{n} \cdot \bar{N}$ & $\begin{array}{lll}\bar{N} & \frac{n}{N}\end{array}$ & $=$ & $\begin{array}{l}\infty \\
- \\
-\end{array}$ & : & S. & ? & $?$ & 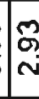 & $\sigma$ & 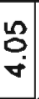 & $\mathrm{v}$ & & mat & $\tilde{\dot{\sigma}}$ & & & 告 \\
\hline & $=\frac{0}{0}$ & $\begin{array}{l}= \\
\overline{0} \\
0\end{array}$ & 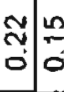 & $\begin{array}{l}0 \\
0 \\
0\end{array}$ & $\stackrel{\sim}{0}$ & 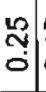 & & $\tilde{s}$ & $\frac{7}{6}$ & $=2$ & & 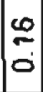 & $\begin{array}{l}\mathbf{0} \\
0 \\
0 \\
\end{array}$ & $\frac{ \pm}{5}$ & $\hat{c}$ & (2) & : & 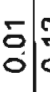 & & ن \\
\hline 음 & $\sigma^{2}$ & $\left(\begin{array}{lll}\infty & 0 \\
\infty & \infty\end{array}\right.$ & \begin{tabular}{l|l}
$\stackrel{\infty}{\infty}$ \\
\end{tabular} & $\underset{N}{*}$ & $\mid \begin{array}{l}8 \\
0 \\
0\end{array}$ & & $\frac{\tilde{m}}{\dot{\omega}}$ & 5 & 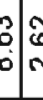 & $\vec{v}=$ & & $\left|\begin{array}{l}R \\
0 \\
0\end{array}\right|$ & $\begin{array}{l}n \\
0 \\
0\end{array}$ & \begin{tabular}{c}
$\approx$ \\
\hdashline \\
0
\end{tabular} & & & $\left|\begin{array}{l}- \\
\infty \\
0\end{array}\right|$ & $\begin{array}{l}\mathscr{m} \\
0 \\
0\end{array}$ & & ${ }_{0}^{-\infty}$ \\
\hline :్తి & $\circ \mid \begin{array}{l}- \\
m \\
0 \\
0\end{array}$ & $\stackrel{0}{0}$ & $\left.\stackrel{8}{\rightarrow}\right|^{8}$ & 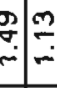 & 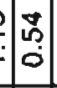 & $\mid$ & की & 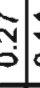 & 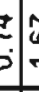 & 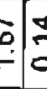 & & זִ & 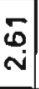 & 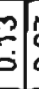 & $\underbrace{5}_{5}$ & & $\mid \begin{array}{l}0 \\
\dot{0}\end{array}$ & $\begin{array}{c}\mathbb{v} \\
\mathbb{N}\end{array}$ & & ì \\
\hline 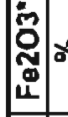 & $\mid \stackrel{\sim}{ }$ & 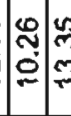 & 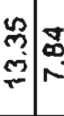 & & 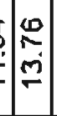 & : & 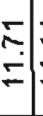 & $\bar{E}$ & & $\stackrel{0}{:}$ & & 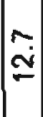 & $\begin{array}{l}\overleftarrow{\infty} \\
\dot{\sigma} \\
\dot{\sigma}\end{array}$ & & & & $\mid \begin{array}{c}\tilde{b} \\
\tilde{n} \\
\tilde{n}\end{array}$ & $\stackrel{m}{?}$ & & \\
\hline | & $\begin{array}{l}0 \\
0 \\
0\end{array}$ & $\begin{array}{ll}\bar{a} \\
\dot{v} \\
\dot{v}\end{array}$ & 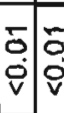 & $\begin{array}{lll}0 \\
0 \\
0\end{array}$ & {$\left[\begin{array}{l}- \\
0 \\
0 \\
8\end{array}\right.$} & $\begin{array}{l}- \\
\vdots \\
0 \\
0\end{array}$ & $\begin{array}{l}- \\
\\
0 \\
\end{array}$ & $\begin{array}{c}0 \\
\dot{0} \\
\bar{v}\end{array}$ & 5 & & & $\mid$\begin{tabular}{|}
$\overline{0}$ \\
0 \\
$\bar{v}$ \\
\end{tabular} & 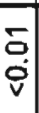 & 5 & : & & $\mid \begin{array}{c}- \\
0 \\
0 \\
\nabla\end{array}$ & $\begin{array}{l}\overline{0} \\
\dot{0} \\
\dot{0}\end{array}$ & & i \\
\hline 엉 & 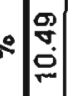 & $\frac{\bar{N}}{\square}$ & \begin{tabular}{c|l}
10 \\
\hdashline \\
0 \\
0
\end{tabular} & 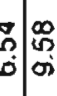 & $\mid \begin{array}{l}z \\
0 \\
0 \\
0\end{array}$ & $\begin{array}{l}g \\
g \\
\sigma^{\circ}\end{array}$ & in & 8 & $\tilde{v}$ & & & & 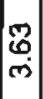 & 1 & & & $=$ & $\overrightarrow{0}$ & & \\
\hline 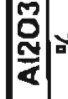 & $\mid \begin{array}{l}0 \\
m \\
\dot{m}\end{array}$ & $\mid \begin{array}{l}n \\
\hat{n} \\
n\end{array}$ & $\mid$ & & {$\left[\begin{array}{l}0 \\
0 \\
2 \\
2\end{array}\right.$} & $\mid \begin{array}{l}\mathfrak{p} \\
\tilde{u} \\
\underline{\sim}\end{array}$ & 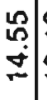 & & 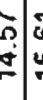 & & & & $\left|\begin{array}{|l}\tilde{N} \\
\infty \\
\omega \\
\end{array}\right|$ & & & & $\mid$ & 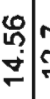 & & \\
\hline 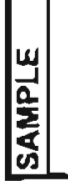 & 豙 & 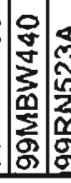 & 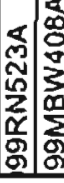 & $\sum_{\substack{0 \\
0}}^{0}$ & 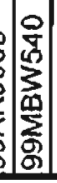 & 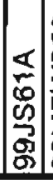 & $\mid$ & 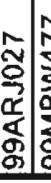 & 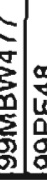 & 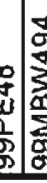 & $\sum_{\substack{0\\
}}^{\infty}$ & 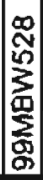 & 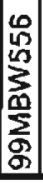 & & 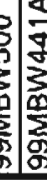 & & 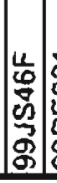 & 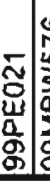 & 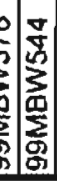 & 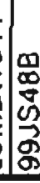 \\
\hline
\end{tabular}


Table 5. Detection limits for geochemical analyses. Analytical methods include: FA-AAS = Fire Assay - Atomic Absorption Spectoscopy, ICP-AES = Inductively Coupled Plasma - Atomic Ernission Spectroscopy, ICP-" MS = Inductively Coupled Plasma - Mass Spectroscopy, AAS = Atomic Absorption Spectroscopy.

\begin{tabular}{|c|c|c|c|c|}
\hline Element & Units & $\begin{array}{c}\text { Lower } \\
\text { Detection } \\
\text { Limir }\end{array}$ & $\begin{array}{c}\text { Ueper } \\
\text { Detection } \\
\text { Limir }\end{array}$ & $\begin{array}{c}\text { Analytical } \\
\text { Merhod }\end{array}$ \\
\hline $\mathrm{Au}$ & $\mathrm{pob}$ & 5 & 10.000 & FA-AAS \\
\hline $\mathrm{Ag}$ & $\mathrm{ppm}$ & 0.2 & 100 & ICP-AES \\
\hline $\mathrm{Ag}^{*}$ & $\mathrm{ppm}$ & 0.02 & 100 & ICP-MS \\
\hline $\mathrm{Ag}^{n}$ & opt & 0.1 & 1.000 & FA-AAS \\
\hline Al & percent & 0.01 & 15 & ICP-AES \\
\hline$A \mathrm{Al}$ & percent & 0.01 & 15 & ICP-MS \\
\hline As & $\mathrm{ppm}$ & 2 & 10.000 & ICP-AES \\
\hline As" & $\mathrm{ppm}$ & 0.2 & 10.000 & ICP-MS \\
\hline $\mathrm{B}$ & ppm & 10 & 10,000 & ICP-AES \\
\hline$B^{*}$ & pprn & 10 & 10.000 & ICP-MS \\
\hline $\mathrm{Ba}$ & gpm & 10 & 10.000 & ICP-AES \\
\hline $\mathrm{Ba}$ & $\mathrm{ppm}$ & 10 & 10.000 & ICP-MS \\
\hline $\mathrm{Be}$ & ppm & 0.5 & 100 & ICP-AES \\
\hline $\mathrm{Be}$ & gpm & 0.5 & 100 & ICP-MS \\
\hline $\mathrm{Bi}$ & $\mathrm{gpm}$ & 2 & 10.000 & ICP-AES \\
\hline $\mathrm{Bi}^{*}$ & $\mathrm{ppm}$ & 0.02 & 10.000 & ICP-MS \\
\hline$B i^{n}$ & ppm & 0.1 & 1.000 & AAS \\
\hline $\mathrm{Ca}$ & percent & 0.01 & 15 & ICP-AES \\
\hline $\mathrm{Ca}$ & percent & 0.01 & 15 & ICP-MS \\
\hline $\mathrm{Cd}$ & ppm & 0.5 & 500 & ICP-AES \\
\hline $\mathrm{Cd}^{*}$ & ppm & 0.1 & 500 & ICP-MS \\
\hline Co & ppm & 1 & 10.000 & ICP-AES \\
\hline $\mathrm{Co}^{*}$ & $\mathrm{ppm}$ & 1 & 10.000 & ICP-MS \\
\hline $\mathrm{Cr}$ & ppm & 1 & 10,000 & ICP-AES \\
\hline $\mathrm{Cr}^{*}$ & $\mathrm{ppm}$ & 1 & 10.000 & ICP-MS \\
\hline $\mathrm{Cu}$ & $\mathrm{ppm}$ & 1 & 10.000 & ICP-AES \\
\hline $\mathrm{Cu}^{*}$ & ppm & 0.2 & 10.000 & ICP-MS \\
\hline $\mathrm{Cu}^{\prime \prime}$ & percent & 0.01 & 100 & AAS \\
\hline $\mathrm{Fe}$ & percent & 0.01 & 15 & ICP-AES \\
\hline $\mathrm{Fe}^{*}$ & percent & 0.01 & 15 & ICP-MS \\
\hline $\mathrm{Ga}$ & ppm & 10 & 10.000 & ICP-AES \\
\hline $\mathrm{Ga}^{*}$ & $\mathrm{ppm}$ & 0.1 & 10.000 & ICP-MS \\
\hline $\mathrm{Ge}^{*}$ & ppm & 0.1 & 500 & ICP-MS \\
\hline $\mathrm{Hg}$ & $\mathrm{ppm}$ & 1 & 10.000 & ICP-AES \\
\hline $\mathrm{Hg}^{*}$ & $\mathrm{ppm}$ & 0.01 & 10.000 & ICP-MS \\
\hline $\mathrm{Hg}^{\prime \prime}$ & $\mathrm{ppm}$ & 0.01 & 100 & AAS \\
\hline $\mathrm{K}$ & percent & 0.01 & 10 & ICP-AES \\
\hline $\mathrm{K}^{*}$ & percent & 0.01 & 10 & ICP-MS \\
\hline
\end{tabular}

\begin{tabular}{|c|c|c|c|c|}
\hline Element & Units & $\begin{array}{l}\text { Lower } \\
\text { Detection } \\
\text { Linnit }\end{array}$ & $\begin{array}{l}\text { Upper } \\
\text { Detection } \\
\text { Limit }\end{array}$ & $\begin{array}{l}\text { Analytical } \\
\text { Method }\end{array}$ \\
\hline $\mathrm{La}$ & ppm & 10 & 10,000 & ICP-AES \\
\hline$\overline{L a *}$ & ppm & 10 & 10,000 & ICP-MS \\
\hline $\mathrm{Mg}$ & percent & 0.01 & 15 & ICP-AES \\
\hline $\mathrm{Mg}$ & percent & 0.01 & 15 & ICP-MS \\
\hline $\mathrm{Mn}$ & ppm & 5 & 10,000 & ICP-AES \\
\hline $\mathrm{Mn}$ & gpm & 5 & 10,000 & ICP-MS \\
\hline Mo & $\mathrm{ppm}$ & 1 & 10,000 & ICP-AES \\
\hline Mo* & ppm & 0.2 & 10,000 & ICP-MS \\
\hline $\mathrm{Na}$ & percent & 0.01 & 10 & ICP-AES \\
\hline $\mathrm{Na}^{*}$ & percent & 0.01 & 10 & ICP-MS \\
\hline $\mathrm{Ni}$ & ppm & 1 & 10,000 & ICP-AES \\
\hline $\mathrm{Ni}=$ & ppm & 1 & 10,000 & ICP-MS \\
\hline $\mathrm{P}$ & ppm & 10 & 10,000 & ICP-AES \\
\hline $\mathrm{P}$ & $\mathrm{gpm}$ & 10 & 10,000 & ICP-MS \\
\hline $\mathrm{Pb}$ & ppm & 2 & 10,000 & ICP-AES \\
\hline $\mathrm{Pb}^{*}$ & ppm & 2 & 10,000 & ICP-MS \\
\hline $\mathrm{S}$ & percent & 0.01 & 5 & ICP-AES \\
\hline$S^{*}$ & percent & 0.01 & 5 & ICP-MS \\
\hline $\mathrm{Sb}$ & $\mathrm{ppm}$ & 2 & 1 & ICP-AES \\
\hline $\mathrm{Sb}^{*}$ & ppm & 0.1 & 10,000 & ICP-MS \\
\hline $\mathrm{Sc}$ & ppm & 1 & 10,000 & ICP-AES \\
\hline $\mathrm{Se}^{*}$ & ppm & 1 & 10,000 & ICP-MS \\
\hline$S_{n}$ & ppm & 2 & 1000 & AAS \\
\hline $\mathrm{S} r$ & ppm & 1 & 10,000 & ICP-AES \\
\hline $\mathrm{Sr} r^{*}$ & ppm & 1 & 10,000 & ICP.MS \\
\hline $\mathrm{Te}^{*}$ & ppm & 0.1 & 500 & ICP-MS \\
\hline $\mathrm{Ti}$ & percent & 0.01 & 10 & ICP-AES \\
\hline $\mathrm{Ti}^{*}$ & percent & 0.01 & 10 & ICP-MS \\
\hline $\mathrm{Tl}$ & ppm & 10 & 10,000 & ICP-AES \\
\hline$T 1^{*}$ & $\mathrm{PPm}$ & 0.1 & 10,000 & ICP-MS \\
\hline $\mathrm{U}$ & $\mathrm{ppm}$ & 10 & 10,000 & ICP-AES \\
\hline$U *$ & ppm & 0.05 & 10,000 & ICP-MS \\
\hline $\mathrm{V}$ & ppm & 1 & 10,000 & ICP-AES \\
\hline$V *$ & $\mathrm{ppm}$ & 0.05 & 10.000 & ICP-MS \\
\hline$W$ & ppm & 10 & 10,000 & 1CP-AES \\
\hline$W *$ & ppm & 0.05 & 10,000 & [CP-MS \\
\hline $2 n$ & ppm & 2 & 10,000 & ICP-AES \\
\hline $\mathrm{Zn}^{*}$ & ppm & 2 & 10,000 & ICP-MS \\
\hline
\end{tabular}


ICP- 
Table 6. Detection limits for " $83 \mathrm{Hn}^{\prime \prime}$ geochemical analyses. Analytical methods include: AAS = Atomic Absorption Spectroscopy; XRF = X-ray Fluorescence.

\begin{tabular}{|l|c|c|c|c|}
\hline Element & Units & $\begin{array}{c}\text { Lower } \\
\text { Detecrion } \\
\text { Limit }\end{array}$ & $\begin{array}{c}\text { Upper } \\
\text { Detection } \\
\text { Limit }\end{array}$ & $\begin{array}{c}\text { Aralytical } \\
\text { Method }\end{array}$ \\
\hline $\mathrm{Au}$ & $\mathrm{ppb}$ & 5 & $?$ & AAS \\
\hline $\mathrm{Ag}$ & $\mathrm{ppm}$ & 0.2 & 100 & AAS \\
\hline $\mathrm{As}$ & $\mathrm{ppm}$ & 5 & $?$ & AAS \\
\hline $\mathrm{Ba}$ & $\mathrm{ppm}$ & 20 & $?$ & XRF \\
\hline $\mathrm{Bi}{ }^{n}$ & $\mathrm{ppm}$ & 0.1 & 1,000 & AAS \\
\hline $\mathrm{Co}$ & $\mathrm{ppm}$ & 2 & $?$ & $\mathrm{AAS}$ \\
\hline $\mathrm{Cu}$ & $\mathrm{ppm}$ & 5 & $?$ & $\mathrm{AAS}$ \\
\hline $\mathrm{Hg}$ & $\mathrm{ppm}$ & 0.01 & 100 & AAS \\
\hline $\mathrm{Mn}$ & $\mathrm{ppm}$ & 1 & $?$ & AAS \\
\hline $\mathrm{Mo}$ & $\mathrm{ppm}$ & 1 & $?$ & AAS \\
\hline $\mathrm{Ni}$ & $\mathrm{ppm}$ & 1 & $?$ & AAS \\
\hline $\mathrm{Pb}$ & $\mathrm{ppm}$ & 2 & $?$ & AAS \\
\hline $\mathrm{Sb}$ & $\mathrm{ppm}$ & 2 & $?$ & AAS \\
\hline $\mathrm{Sn}$ & $\mathrm{ppm}$ & 2 & 1000 & AAS \\
\hline $\mathrm{W}$ & $\mathrm{ppm}$ & 1 & $?$ & Colomerric rechnique \\
\hline $\mathrm{Zn}$ & $\mathrm{ppm}$ & 2 & $?$ & AAS \\
\hline
\end{tabular}


Table 7. Detection limits for major-oxide. minor oxide, and trace element analyses. Analytical methods include: $X R F=X-R a y$ Fluorescence Spectroscopy, and AAS $=$ Atomic Absorption Spectroscopy. Note: LOI* $=$ Loss On Ignition.

\begin{tabular}{|c|c|c|c|c|}
\hline Element & Units & $\begin{array}{c}\text { Lower } \\
\text { Detection } \\
\text { Limir }\end{array}$ & $\begin{array}{c}\text { Upper } \\
\text { Detection } \\
\text { Limit }\end{array}$ & $\begin{array}{l}\text { Analytical } \\
\text { Method }\end{array}$ \\
\hline $\mathrm{Al}_{2} \mathrm{O}_{3}$ & percent & 0.01 & 100.00 & $\mathrm{XRF}$ \\
\hline $\mathrm{CaO}$ & percent & 0.01 & 100.00 & $\mathrm{XRF}$ \\
\hline $\mathrm{Cr}_{2} \mathrm{O}_{3}$ & percent & 0.01 & 100.00 & XRF \\
\hline $\mathrm{Fe}_{2} \mathrm{O}_{3}^{*}$ & percent & 0.01 & 100.00 & $\mathrm{XRF}$ \\
\hline $\mathrm{K}_{2} \mathrm{O}$ & percent & 0.01 & 100.00 & XRF \\
\hline $\mathrm{MgO}$ & percent & 001 & 100.00 & XRF \\
\hline $\mathrm{MnO}$ & percent & 0.01 & 100.00 & XRF \\
\hline $\mathrm{Na}_{2} \mathrm{O}$ & percent & 0.01 & 100.00 & XRF \\
\hline $\mathrm{P}_{2} \mathrm{O}_{5}$ & percent & 0.01 & 100.00 & XRF \\
\hline $\mathrm{SiO}_{2}$ & percent & 0.01 & 100.00 & $\mathrm{XRF}$ \\
\hline $\mathrm{TiO}_{2}$ & percent & 0.01 & 100.00 & XRF \\
\hline $\mathrm{LOI}^{*}$ & percent & 0.01 & 100.00 & $\mathrm{XRF}$ \\
\hline Total & percent & 0.01 & 105.00 & Calculation \\
\hline $\mathrm{Ba}$ & $\mathrm{gpm}$ & 5 & 50,000 & XRF \\
\hline $\mathrm{Rb}$ & $\mathrm{ppm}$ & 2 & 50,000 & XRF \\
\hline $\mathrm{Sr}_{\Gamma}$ & $\mathrm{ppm}$ & 2 & 50,000 & $\mathrm{XRF}$ \\
\hline $\mathrm{Nb}$ & PPm & 2 & 50,000 & XRF \\
\hline $\mathrm{Z}_{\mathrm{I}}$ & $\mathrm{ppm}$ & 3 & 50,000 & $\mathrm{XRF}$ \\
\hline$Y$ & gpm & 2 & 50,000 & XRF \\
\hline $\mathrm{CL}$ & $\mathrm{ppm}$ & 1 & 10,000 & AAS \\
\hline $\mathrm{Ni}$ & $\mathrm{ppm}$ & 1 & 10,000 & AAS \\
\hline $\mathrm{Cr}$ & $\mathrm{ppm}$ & 2 & 10,000 & AAS \\
\hline
\end{tabular}

